\title{
A Neuropsin-based Optogenetic Tool for Precise Control of
}

\section{$\mathbf{G}_{\mathrm{q}}$ signaling}

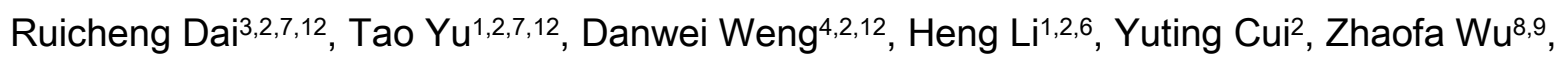

Qingchun Guo ${ }^{10,5}$, Haiyue Zou11,5, Wenting Wu'1,2,7, Xinwei Gao5 ${ }^{5}$ Zhongyang Qi², Yuqi Ren², Shu

Wang ${ }^{5}$, Yulong Li $i^{8,9}$, Minmin Luo $2,4,5,6,13^{*}$

${ }^{1}$ School of Life Sciences, Tsinghua University, Beijing 100084, China

${ }^{2}$ National Institute of Biological Sciences (NIBS), Beijing 102206, China

${ }^{3}$ School of Life Sciences, Peking University, Beijing 100871, China

${ }^{4}$ Graduate School of Peking Union Medical College, Beijing 100730, China

${ }^{5}$ Chinese Institute for Brain Research, Beijing 102206, China

${ }^{6}$ Tsinghua Institute of Multidisciplinary Biomedical Research (TIMBR), Beijing 102206, China

${ }^{7}$ Peking University-Tsinghua University-NIBS Joint Graduate Program, NIBS, Beijing 102206,

China

${ }^{8}$ State Key Laboratory of Membrane Biology, Peking University School of Life Sciences, Beijing 100871, China

9PKU-McGovern Institute for Brain Research, Beijing 100871, China

${ }^{10}$ Capital Medical University, Beijing 102206, China

${ }^{11}$ Academy for Advanced Interdisciplinary Studies, Peking University, Beijing 100871, China

12These authors contributed equally 
bioRxiv preprint doi: https://doi.org/10.1101/2022.02.22.481462; this version posted February 23, 2022. The copyright holder for this preprint (which was not certified by peer review) is the author/funder, who has granted bioRxiv a license to display the preprint in perpetuity. It is made available under aCC-BY-NC-ND 4.0 International license.

${ }^{13}$ Lead contact

${ }^{*}$ Correspondence: luominmin@nibs.ac.cn 


\section{Abstract}

$\mathrm{G}_{\mathrm{q}}$-coupled receptors regulate numerous physiological processes by activating enzymes and inducing intracellular $\mathrm{Ca}^{2+}$ signals. There is a strong need for an optogenetic tool that enables powerful experimental control over $\mathrm{G}_{\mathrm{q}}$ signaling. Here, we present chicken opsin 5 (cOpn5) as the long sought-after, single-component optogenetic tool that mediates ultra-sensitive optical control of intracellular $G_{q}$ signaling with high temporal and spatial resolution. Expressing cOpn5 in mammalian cells enables blue light-triggered, $\mathrm{G}_{\mathrm{q}}$-dependent $\mathrm{Ca}^{2+}$ release from intracellular stores and protein kinase $\mathrm{C}$ activation. Strong $\mathrm{Ca}^{2+}$ transients were evoked by brief light pulses of merely 10 ms duration and at 3 orders lower light intensity of that for common optogenetic tools. Photostimulation of cOpn5-expressing cells at the subcellular and single-cell levels generated intracellular and intercellular $\mathrm{Ca}^{2+}$ wave propagation, respectively, thus demonstrating the high spatial precision of cOpn5 optogenetics. The cOpn5-mediated optogenetics could also be applied to activate neurons and control animal behavior in a circuit-dependent manner. We further revealed that optogenetic activation of copn5-expressing astrocytes induced massive ATP release and modulation neuronal activation in the brain of awake, behaving mice. copn5 optogenetics may find broad applications in studying the mechanisms and functional relevance of $G_{q}$ signaling in both non-excitable cells and excitable cells in all major organ systems.

\section{Main}

G-protein-coupled receptors (GPCRs) modulate many intracellular signaling pathways and represent some of the most intensively studied drug targets ${ }^{1}$. Upon ligand binding, the GPCR undergoes a conformation change that is transmitted to heterotrimeric $\mathrm{G}$ proteins, which are multi-subunit complexes comprising $G_{\alpha}\left(G_{q / 11}, G_{s}, G_{i / o}\right.$ and $\left.G_{12 / 13}\right)$ and tightly associated $\mathrm{G}_{\beta \gamma}$ subunits ${ }^{2}$. The $\mathrm{G}_{q}$ proteins, a subfamily of $\mathrm{G}_{\alpha}$ subunits, couple to a class of GPCRs to 
mediate cellular responses to neurotransmitters, sensory stimuli, and hormones throughout the body $^{3,4}$. Their primary downstream signaling targets include phospholipase C beta (PLC- $\beta$ ) enzymes, which catalyze the hydrolysis of phospholipid phosphatidylinositol bisphosphate $\left(\mathrm{PIP}_{2}\right)$ into inositol trisphosphate $\left(\mathrm{IP}_{3}\right)$ and diacylglycerol (DAG). $\mathrm{IP}_{3}$ triggers intracellular $\mathrm{Ca}^{2+}$ release, which together with DAG activates protein kinase $C(P K C)^{5}$. Studies have demonstrated that the activation of $\mathrm{G}_{\mathrm{q}}$-coupled receptors often produces rapid (within seconds) and robust increase in $\mathrm{Ca}^{2+}$ signals ${ }^{6}$. Given that $\mathrm{Ca}^{2+}$ signals and PKC activity impact nearly every cellular process in both non-excitable cells and excitable cells, developing tools that precisely control intracellular $\mathrm{G}_{\mathrm{q}}$ signaling would be highly valuable for studying the mechanisms and physiological functions of $\mathrm{G}_{\mathrm{q}}$-coupled receptors.

Optogenetics uses light-responsive proteins to achieve optically-controlled perturbation of cellular activities with genetic specificity and high spatiotemporal precision ${ }^{7,8}$. Since the early discoveries of optogenetic tools using light-sensitive ion channels and transporters, diverse technologies have been developed and now support optical interventions into intracellular second messengers, protein interactions and degradation, and gene transcription ${ }^{9-12}$. Optogenetic tools that control $G_{q}$ signaling may have several advantages over current approaches including chemogenetics and photoactivatable small molecules ${ }^{13-15}$ : unlike chemogenetics, they potentially offer subsecond temporal resolution and subcellular spatial resolution; unlike photoactivatable compounds, they provide control of genetically-identified cell types in complex organ systems. However, despite several intensive efforts, to date there has been few optogenetic tool that enables rapid and effective activation of $\mathrm{G}_{\mathrm{q}}$ signaling ${ }^{16-18}$. Opto-a1AR, a creatively designed $\mathrm{G}_{\mathrm{q}}$-coupled rhodopsin-GPCR chimera, induces mild intracellular $\mathrm{Ca}^{2+}$ increase only after long-time (>60 s) photostimulation ${ }^{19}$. Melanopsin (Opn4) in a subset of mammalian retinal ganglion cells is a $G_{q}$-coupled opsin that mediates no-image-forming visual functions $\mathrm{s}^{20-24}$. However, cells expressing mOpn4L show weak $\mathrm{Ca}^{2+}$ 
responses even after prolonged (15-60 s) exposure to bright light illumination, and require continuous chromophore addition in the culture medium ${ }^{25-27}$. Indeed, systematic characterizations have revealed major limitations of these two tools for in vitro and in vivo applications $^{28-30}$. Opto-a1AR and Opn4 thus only partially mimick the activation of endogenous $\mathrm{G}_{\mathrm{q}}$-coupled receptors.

We asked whether some naturally occurring photoreceptors could serve as efficient optogenetic tools for $\mathrm{G}_{\mathrm{q}}$ signaling. Most animals detect light using GPCR-based photoreceptors, which comprise a protein moiety (opsin) and a vitamin A derivative (retinal) that functions as both a ligand and a chromophore ${ }^{31}$. Several thousand opsins have been identified to date ${ }^{32,33}$, and two recent studies reported $G_{i}$-based opsins from mosquito and lamprey for presynaptic inhibition in neurons $^{34,35}$. Opn5 (neuropsin) and its orthologs in many vertebrates have been reported as an ultraviolet (UV)-sensitive opsin that couples to $G_{i}$ proteins $^{36-39}$. Interestingly, exposure to blue light induces an increase in intracellular $\mathrm{Ca}^{2+}$ levels within avian primary Müller glial cells endogenously expressing Opn $5^{40}$, hinting the possibility that certain Opn5 might also couple to $\mathrm{G}_{\mathrm{q}}$ proteins $^{41,42}$.

Here we report that the chicken Opn5 (cOpn5 for simplicity), but not two of its mammalian orthologs, sensitively and strongly mediated blue light-induced activation of $\mathrm{G}_{\mathrm{q}}$ signaling in mammalian cells. Detailed characterizations of cOpn5 reveal that it is at least 3 orders of magnitude higher in light sensitivity and temporal precision than existing $\mathrm{G}_{\mathrm{q}}$-coupled opsin-based tools - opto-a1AR and Opn4, provides subcellular spatial resolution, and does not require chromophore addition. We further demonstrate cOpn5 optogenetics as a highly effective approach for activating neurons to produce robust behavior changes in freely moving mice, as well as for activating astrocytes to induce massive ATP release in vivo. These findings establish that copn5 can be utilized as powerful, single-component optogenetic tools to support experimental investigations into the mechanisms and functions associated with $\mathrm{G}_{\mathrm{q}}$ signaling in 
both non-excitable cells and excitable cells.

\section{Results}

\section{cOpn5 mediates optogenetic activation of $G_{q}$ signaling}

We tested whether heterologous expression of the Opn5 orthologs from chicken, humans, and mice (which share $80-90 \%$ protein sequence identity) have the capacity to mediate blue light-induced $\mathrm{G}_{\mathrm{q}}$ signaling activation within HEK 293T cells (Fig. 1a and Supplementary Table 1). The chicken Opn5 (cOpn5) was co-localized with the EGFP-CAAX membrane maker, indicating that it was effectively expressed on the plasma membrane (Fig. 1b). We used blue light for stimulation and the red intracellular $\mathrm{Ca}^{2+}$ indicator Calbryte ${ }^{\mathrm{TM}} 630 \mathrm{AM}$ dye to monitor the relative $\mathrm{Ca}^{2+}$ response (Fig. 1c; See Supplementary Table 2 for the list of resources). cOpn5 mediated an immediate and strong light-induced increase in $\mathrm{Ca}^{2+}$ signal $\left(-3-\right.$ fold increase in $\mathrm{Ca}^{2+}$ indicator fluorescence intensity relative to its resting fluorescence intensity; abbreviated as $\Delta F / F$ ), whereas no light effect was observed from cells expressing the human or mouse Opn5 orthologs (Fig. 1d; Video 1). Note that we did not supply any exogenous retinal to the culture media, which suggested that endogenous retinal is sufficient to render copn5 functional ${ }^{43}$. Thus, unlike mammalian Opn5, cOpn5 allows effective optical activation of intracellular $\mathrm{Ca}^{2+}$ signals in the human HEK 293T cells.

We then examined whether cOpn5 truly mediated the activation of $\mathrm{G}_{\mathrm{q}}$ signaling, which triggers signal cascades that produce two second messengers: $\mathrm{IP}_{3}$ leading to $\mathrm{Ca}^{2+}$ releases from intracellular stores, and DAG leading to PKC activation. Preincubation of YM-254890, a highly selective $G_{q}$ proteins inhibitor ${ }^{44}$, reversibly abolished the light-induced $\mathrm{Ca}^{2+}$ transients in both cOpn5-expressing cells (Figs. 1 e and $1 \mathrm{f}$ ). We observed strong $\mathrm{Ca}^{2+}$ signals in the absence of extracellular $\mathrm{Ca}^{2+}$, thus indicating $\mathrm{Ca}^{2+}$ release from intracellular stores (Extended Data Fig. 1a). In cOpn5-, but not human OPN5-expressing cells, we also detected a light-induced increase in 
the level of inositol phosphate $\left(\mathrm{IP}_{1}\right)$, the rapid degradation product of $\mathrm{IP}_{3}$; moreover, the extent of this increase was reduced with the treatment of YM-254890 (Fig.1g and Extended Data Fig. 1d; See Supplementary Table 3 for detailed information of statistical analyses). This result thus indicated light-evoked, $\mathrm{G}_{\mathrm{q}}$-dependent $\mathrm{IP}_{3}$ production. In cOpn5-expressing HEK 293T cells, blue light also triggered the phosphorylation of MARCKS protein, a well-established target of PKC ${ }^{45}$, in a PKC activity-dependent manner (Extended Data Figs. 1b and 1c). Consistent with earlier findings indicating that mammalian Opn5 couples to $\mathrm{G}_{i}^{36-38}$, blue light illumination effectively reduced cAMP levels in cells expressing human and mouse Opn5 with retinal addition; however, it had very mild effect in cOpn5-expressing cells in the presence of retinal and had no effect without retinal (Extended Data Fig. 1e). Collectively, these data revealed that blue light illumination enables the efficient coupling of cOpn5, but not its mammalian orthologs, to the $\mathrm{G}_{\mathrm{q}}$ signaling pathway in mammalian cells.

\section{cOpn5-mediated optogenetics is sensitive and precise}

Given that light-stimulated cOpn5 mimics endogenous $\mathrm{G}_{\mathrm{q}}$-coupled GPCRs to rapidly activate the $\mathrm{G}_{\mathrm{q}}$ signaling pathway, we asked whether cOpn5 could serve as the long sought-after optogenetic tool for $G_{q}$ signaling, and more importantly, whether it has features common to other popular optogenetic tools, such as high light sensitivity, single-component convenience, and high spatiotemporal resolution. We first systematically characterized the light-activating properties of cOpn5-expressing HEK 293T cells. Although Opn5 is previously considered as an ultraviolet (UV)-sensitive photoreceptor ${ }^{37}$, mapping with a set of wavelengths ranging $365-630 \mathrm{~nm}$ at a fixed light intensity of $\left(100 \mu \mathrm{W} / \mathrm{mm}^{2}\right)$ revealed that the $470 \mathrm{~nm}$ blue light elicited the strongest $\mathrm{Ca}^{2+}$ transients, with the UVA light (365 and $395 \mathrm{~nm}$ ) being less effective and longer-wavelength visible light (561 nm or above) completely ineffective (Fig. 2a). We then tested the effects of varying photostimulation durations. Stimulating with brief light pulses $(1,5,10,20,50 \mathrm{~ms} ; 16$ 
$\left.\mu \mathrm{W} / \mathrm{mm}^{2} ; 470 \mathrm{~nm}\right)$ showed that the $\mathrm{Ca}^{2+}$ response achieved the saturation mode with light duration over $10 \mathrm{~ms}$. Longer light durations did not further increase the $\mathrm{Ca}^{2+}$ signal amplitude at this light intensity (Fig. 2b). Delivering brief blue light pulses (10 ms; $470 \mathrm{~nm}$ ) at different intensities showed that the light of $\sim 4.8 \mu \mathrm{W} / \mathrm{mm}^{2}$ and $16 \mu \mathrm{W} / \mathrm{mm}^{2}$ produced about half maximum and full maximum responses, respectively (Fig. 2c). The response time courses revealed that 10 ms blue light pulses $\left(16 \mu \mathrm{W} / \mathrm{mm}^{2}\right)$ generated significant $\mathrm{Ca}^{2+}$ signals within $1 \mathrm{~s}$ and produced peak responses within $2.5 \mathrm{~s}$ (Fig. 2c). For $10 \mathrm{~ms}, 16 \mu \mathrm{W} / \mathrm{mm}^{2}$ blue light stimulation, time to $10 \%$ peak activation was $1.36 \pm 0.55 \mathrm{~s}$; time to $90 \%$ peak activation was $2.37 \pm 0.87 \mathrm{~s}$; decay time $\mathrm{\tau}=$ $18.66 \pm 4.98 \mathrm{~s}$. Therefore, the light sensitivity of cOpn5 is 3-4 orders of magnitude higher than the reported values of the light-sensitive $G_{q}$-coupled GPCRs and even 2-3 orders higher than those of the commonly used optogenetic tool Channelrhodopsin-2 (ChR2) ${ }^{46,47}$ (Supplementary Table 4). Together, these results indicate that cOpn5 could function as a single-component optogenetic tool without additional retinal, and that copn5 is super-sensitive to blue light for its full activation requiring low light intensity $\left(16 \mu \mathrm{W} / \mathrm{mm}^{2}\right)$ and short duration (10 ms).

Designer Receptors Exclusively Activated by Designer Drugs (DREADD)-based chemogenetic tools efficiently modulate cellular activity ${ }^{48,49}$. For example, hM3Dq expression allows the activation of $\mathrm{G}_{\mathrm{q}}$ signaling by adding the exogenous small molecule ligand clozapine-N-oxide $(\mathrm{CNO})^{14,50,51}$. We thus compared the $\mathrm{Ca}^{2+}$ signals in response to $10 \mathrm{~s}$ infusion of CNO on hM3Dq-expressing HEK 293T cells to that evoked by $10 \mathrm{~s}$ photostimulation of cOpn5-expressing cells. Although the response amplitudes were similar, cOpn5-mediated optogenetic stimulation produced faster and temporally more precise response, as well as more rapid recovery than hM3Dq-mediated chemogenetic stimulation (Fig. 2b and Extended Data Fig. $2 a-c)$. These results thus indicate that cOpn5-mediated optogenetics are more controllable in temporal accuracy than those of hM3Dq-mediated chemogenetics.

What's more, cOpn5 optogenetics provides the major advantage of spatially precise control 
of cellular activity. Restricting brief light stimulation (63 ms) into individual cOpn5-expressing HEK 293 T cells resulted in the immediate activation of the stimulated cell. Interestingly, in high cell confluence areas, the $\mathrm{Ca}^{2+}$ signals propagated to surrounding cells, thus suggesting intercellular communication among HEK 293T cells through a yet-to-identified mechanism (Fig.

\section{2d, 2e and Supplementary Video 2).}

We directly compared the performance of cOpn5 to those of opto-a1AR and Opn4, which had been proposed for optogenetic control of $G_{q}$ signaling. Following the protocol in a previous report $^{19}$, we found that $15 \mathrm{~s}$ illumination at the rather strong intensity level $\left(7 \mathrm{~mW} / \mathrm{mm}^{2}\right)$ was completely ineffective in opto-a1AR-epxpressing cells; further increasing the light exposure duration to $60 \mathrm{~s}$ triggered a slow and small $(\sim 0.5 \Delta F / F) \mathrm{Ca}^{2+}$ signal increase (Figs. $2 \mathrm{f}$ and $\left.2 \mathrm{~g}\right)$. We also compared the performance of cOpn5 to that of mouse Opn4L, a natural opsin which was reported as a tool for $\mathrm{G}_{\mathrm{q}}$ signaling activation ${ }^{26,52}$. Without the addition of all-trans-retinal (ATR), neither brief blue light pulses $\left(16 \mu \mathrm{W} / \mathrm{mm}^{2}\right.$ ) nor prolonged strong light illumination (25 s, 40 $\mathrm{mW} / \mathrm{mm}^{2}, 470 \mathrm{~nm}$ ) had slightly effect on the $\mathrm{Ca}^{2+}$ signals in mOPN4L-expressing HEK 293T cells (Figs. 2h and 2i). Following the addition of exogenous ATR, long exposure of very strong illumination $\left(25 \mathrm{~s} ; 40 \mathrm{~mW} / \mathrm{mm}^{2}\right)$ triggered a slow $\mathrm{Ca}^{2+}$ signal increase $(\sim 1 \Delta F / F)$ in mOPN4L-expressing cells; by contrast, in cOpn5-expressing cells the light pulses of only 1/2500 duration $(10 \mathrm{~ms})$ and $1 / 2500$ intensity $\left(16 \mu \mathrm{W} / \mathrm{mm}^{2}\right)$ produced nearly 6 -fold increase in $\mathrm{Ca}^{2+}$ signals (Figs. $2 \mathrm{~h}$ and $2 \mathrm{i}$ ). Therefore, compared with existing opsin-based tools (opto-a1AR and mOpn4L), cOpn5 is much more light-sensitive (at least $\sim 3$ orders higher sensitivity), requires much shorter time exposure (10 ms vs. 25 s or $60 \mathrm{~s}$ ), and produces severalfold stronger responses (Supplementary Table 4).

\section{cOpn5 optogenetics activates neurons and modulates animal behaviors}

We explored the application of cOpn5-mediated optogenetics directly in neurons. We first 
examined whether cOpn5 could mediate light-induced $\mathrm{Ca}^{2+}$ signals. Using AAV and the pan-neuronal SYN promoter, we expressed cOpn5 and the red $\mathrm{Ca}^{2+}$ indicator jRGECO1a in mouse cortical neurons (Fig. 3a). In brain slice preparations, application of blue light pulses (10s; $100 \mu \mathrm{W} / \mathrm{mm}^{2} ; 473 \mathrm{~nm}$ ) reliably evoked $\mathrm{Ca}^{2+}$ transients in neurons (Figs. 3b and 3c). Thus, cOpn5 also enables light-induced activation in neurons.

We next assessed the utility of cOpn5-mediated optogenetics for modulating animal behavior. The lateral hypothalamus (LH) participate in reward processing and feeding regulation $^{53-55}$. We expressed cOpn5 in the LH GABAergic neurons of VGAT-Cre mice and implanted optical fibers to deliver light pulses into the LH of freely behaving mice (Fig. 3d and Extended Data Fig. 3b). Consistent with a role of LH GABA neurons in promoting feeding behavior $^{55}$, light stimulation (20 Hz; $5 \mathrm{~ms} /$ pulse; $473 \mathrm{~nm} ; 0.75 \mathrm{~mW}$ output from the fiber tip) elicited a significant increase in food intake in cOpn5-expressing mice but not the EGFP-expressing control mice (Fig. 3e). We also used a food-foraging behavior task to test the effect of cOpn5-mediated optogenetic activation of GABA neurons in the zona incerta (ZI) (Fig. $3 f$ and Extended data Fig. 3c), a region known to drive compulsive eating ${ }^{56}$. cOpn5-expressing mice, but not the EGFP-expressing mice, showed a significantly increase in the time of foraging high fat food pellets upon repeated light stimulation (Fig. 3g). Notably, mice maintained the behavior (feeding behavior or high-fat food foraging behavior) while the light was on, and immediately stopped the behavior when the light was off (Supplementary Video 3). Thus, cOpn5 is effective for rapidly, accurately, and reversibly modulating animal behavioral states.

Finally, we investigated the effect of light-induced cOpn5 activation on the electrophysiological properties of lateral hypothalamus and zona incerta neurons in slice preparations (Extended Data Fig. 3a). Light pulses rapidly drove vigorous firing of action potentials (Figs. 3h and 3i). What's more, we observed two types of activation patterns of cortical and subcortical neurons. In a majority of neurons (17 out 29 neurons), brief light pulses (10 ms) 
rapidly evoked strong inward currents (100-1000 pA) and drove vigorous firing of action potentials (Extended Data Fig. 3b, left and 3c). In the other 12 out of the 29 neurons recorded, blue light pulses $(10 \mathrm{~ms})$ induced a small depolarizing current $(\sim 20 \mathrm{pA})$ in the voltage-clamp mode, and induced delayed-yet-robust firing of action potentials in the current-clamp mode (Extended Data Fig. 3b, right and 3c). Neurons were repeatedly stimulated with 10 ms pulses at $10 \mathrm{~Hz}$, and exhibited a non-attenuated mode in firing rate across repetitive trials of light stimulation (Extended Data Fig. 3d). Of note, unlike those generated by ChR2 optogenetics ${ }^{57}$, the action potentials evoked by cOpn5 photostimulation were not time-locked to light pulses in any of neurons recorded.

\section{cOpn5 optogenetic activation of astrocytes evokes calcium signal}

We extended the findings into primary cell cultures. It was reported transient elevations of calcium concentration occur in astrocytes providing cells with a specific form of excitability ${ }^{58-61}$. We expressed cOpn5 in primary astrocyte cultures prepared from the neonatal mouse brain with AAV vectors for bicistronic expression of cOpn5 and the EGFP marker protein (Fig. 4c). Using the Calbryte ${ }^{\mathrm{TM}} 630 \mathrm{AM}$ dye to monitor $\mathrm{Ca}^{2+}$ levels, we found that blue light illumination of cOpn5-expressing astrocytes produced strong $\mathrm{Ca}^{2+}$ transients ( $\left.\sim \Delta F / F\right)$ (Figs. $4 \mathrm{a}, 4 \mathrm{~b}$ and Supplementary Video 1). Confining the light pulses $(63 \mathrm{~ms})$ to subcellular regions produced $\mathrm{Ca}^{2+}$ signal propagation within the stimulated cells (Fig. 4d). Resembling the tests in HEK 293T cells, we observed wave-like propagation of $\mathrm{Ca}^{2+}$ signals that proceeded from the stimulated astrocyte gradually to more distal, non-stimulated, astrocytes (Figs. 4e, 4f and Supplementary Video 2). This optically-triggered intercellular $\mathrm{Ca}^{2+}$ wave was reminiscent of the dynamics of astrocytic networks that were initially discovered using neurochemical and mechanical stimulation ${ }^{58,59}$.

We next tested the performance of cOpn5-mediated optogenetics in vivo. We carried out cOpn5-mediated optogenetic activation of astrocytes and monitored calcium signal ${ }^{60}$. Specifically, 
we expressed cOpn5 and the GCaMP6s sensor in the mouse S1 sensory cortex following the infusion of $\mathrm{AAV}$ vectors containing the GfaABC1D promoter. (Fig. 4g), which drove gene expression selectively and efficiently in cortical astrocytes ${ }^{62,63}$. We initially expected that, in addition to the $920 \mathrm{~nm}$ light from pulsed laser for two-photon imaging, blue light pulses would be required to stimulate calcium signals. Strikingly, upon the $920 \mathrm{~nm}$ light delivered from microscope, triggered remarkable calcium elevation was observed in the cOpn5- and GCaMP6s-expressing mice but not in the control mice that expressed the mCherry- and GCaMP6s but lacked cOpn5 expression (Figs. 4h-4j and Supplementary Video 4 ). The calcium events were persistent with only slight decrease during 20min constant stimulation (Extended Data Fig. 4a). Together, these experiments demonstrate that cOpn5 optogenetics allows precise spatiotemporal control of $\mathrm{G}_{\mathrm{q}}$ signaling with millisecond and subcellular resolutions.

\section{cOpn5 optogenetic activation of astrocytes induces massive ATP release and regulates neuron activation in vivo}

Astrocytes represent an important population of non-excitable cells in the central nervous system and are known to regulate a variety of processes, including neurogenesis and synaptogenesis, blood-brain barrier permeability, and extracellular homeostasis ${ }^{64-68}$. However, to date direct optogenetic control of astrocytes has achieved only limited success ${ }^{28,69,70}$. ATP is known as a key messenger for inter-astrocyte communication ${ }^{71-73}$. In cell cultures and brain slices, ATP can be released through hemichannels in a $\mathrm{Ca}^{2+}$-independent manner or through vesicle release in a $\mathrm{Ca}^{2+}$-dependent manner ${ }^{74,75}$. It has remained controversial whether intracellular $\mathrm{Ca}^{2+}$ signals in astrocyte induce ATP release in vivo, and if so, how such release manifests in real-time. We carried out cOpn5-mediated optogenetic activation of astrocytes and monitored ATP release using the recently-developed GPCR Activation-Based ATP sensor $\mathrm{GRAB}_{\text {ATP }}{ }^{76,77}$. Specifically, we expressed cOpn5 and the GRAB ${ }_{\text {ATP }}$ sensor in the mouse S1 
sensory cortex following the infusion of AAV vectors containing the GfaABC1D promoter (Fig. 5a).We tested the gene was selectively and efficiently expressed in cortical astrocytes (Figs. 5b and $5 c)^{62,63}$. We also used $920 \mathrm{~nm}$ light from pulsed laser for two-photon imaging. The $920 \mathrm{~nm}$

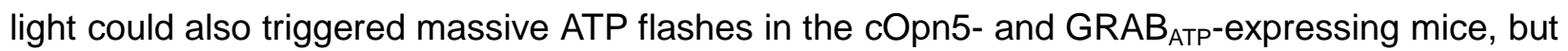
not in mice that expressed the ATP sensor but lacked cOpn5 expression (Fig. 5e). Individual ATP flashes typically ranged in diameters of 20-100 $\mu \mathrm{m}$ and lasted for $\sim 1$ min (Supplementary Video 5). The flash frequency gradually increased following $\sim 1 \mathrm{~min}$ of initial quiescence and up to the level of $\sim 50$ flashes per min within the imaging area $\left(636 \times 636 \mu \mathrm{m}^{2}\right)$ in $\sim 5 \mathrm{~min}$ (Figs. $5 \mathrm{~d}$ and $\left.5 f\right)$. In mice expressing $\mathrm{GRAB}_{\text {ATP }}$ alone, we observed sporadic ATP events ( $\sim .3$ flashes per min within the imaging area; Figs. $5 e$ and $5 \mathrm{~g}$ ). The observed ATP flash frequency in cOpn5-expressing mice was $\sim 1300$ times more than non-cOpn5-expressing mice (Figs. 5e, $5 \mathrm{~g}$ and Supplementary Video 5). Moreover, high-frequency ATP flashes also occurred in the repeated trials (Extended Data Fig. 4b). Given that hM3Dq allows the activation of astrocytes with $\mathrm{Ca}^{2+}$ elevation by $\mathrm{CNO}^{60,78}$, we also performed ATP imaging 40 min following the treatment of intraperitoneal injections of CNO into $\mathrm{GRAB}_{\text {ATP- }}$ hM3Dq expressing mice that lacked cOpn5 expression. Following the CNO treatments, the ATP flash events were approximately equal to the basal condition (Figs. 5e and $5 \mathrm{~g}$ and Supplementary Video 5) and hM3Dq was ineffectively to trigger astrocytes activation-dependent ATP release. These experiments thus demonstrate that copn5-mediated optogenetic activation of $G_{q}$ signaling in astrocytes is particularly effective in inducing ATP release in vivo in the unique form of ATP flashes.

Astrocytes release several gliotransmitters, which together with their metabolites, exert complex modulatory effects on synaptic activity, neuronal oscillation, and various animal behaviors $^{79,80}$. For example, the gliotransmitters ATP and glutamate can directly activate nearby neurons $^{64,72,81,82}$, whereas adenosine, the major metabolite of ATP, may inhibit neurons through the A1-type adenosine receptor ${ }^{83}$. It has remained elusive how astrocyte activation affects the 
activity of individual neurons in behaving animals. We expressed cOpn5 in astrocytes and the green $\mathrm{Ca}^{2+}$ indicator GCaMP7 in pyramidal neurons within the S1 cortical area. We then carried out two-photon imaging of neuronal $\mathrm{Ca}^{2+}$ signals in response to copn5-mediated optical activation of astrocytes in head-fixed awake, behaving mice (Fig. 5h). Initially neurons were largely quiescent, and then exhibited a gradual increase in the frequency and amplitudes of $\mathrm{Ca}^{2+}$ transients in approximately 5 min (Fig. 5i). Group data from a total of 193 neurons revealed that photostimulation of cOpn5-expressing significantly increased the frequency of $\mathrm{Ca}^{2+}$ transients and the in neurons. MCherry-expressing control group showed no influence on the decoded spiking rates of neurons or even slightly decrease the $\mathrm{Ca}^{2+}$ transients frequency (Figs. 5i, 5j and Extended Data Figs. 4c, 4d), suggesting that activating $G_{q}$ signaling in astrocytes produces an overall excitatory effect on nearby cortical neurons in behaving state. Both astrocytic ATP imaging and neuronal $\mathrm{Ca}^{2+}$ imaging demonstrated that the long-wavelength $(920 \mathrm{~nm})$ light from pulsed laser for two-photon imaging is able to activate cOpn5, indicating the possibility of two-photon optogenetics for cOpn5.

\section{Discussion}

Here, we demonstrate the use of cOpn5 as an extremely effective optogenetic tool for activating $\mathrm{G}_{\mathrm{q}}$ signaling. Previous studies have characterized mammalian Opn5 as a UV-sensitive $\mathrm{G}_{\mathrm{i}}$-coupled opsin; we present the surprising finding that visible blue light can induce rapid $\mathrm{Ca}^{2+}$ transients, $\mathrm{IP}_{1}$ accumulation, and $\mathrm{PKC}$ activation in cOpn5-expressing mammalian cells. cOpn5 in mouse astrocytes effectively mediates light-evoked ATP release and elevates neuron activity in vivo. We also show that copn5 allows optical activation of neurons and precise control of animal behaviors. Importantly, copn5 is a powerful yet easy-to-use, single-component system that does not require an exogenous chromophore. We envision that cOpn5-based optogenetics will be an enabling technique for investigating the important physiological and behavioral 
functions regulated by $G_{q}$ signaling in both non-excitable and excitable cells.

Supplementary Table 4 lists the key features of cOpn5 by directly comparing its response amplitudes, light sensitivity, temporal resolution, and the requirement of additional chromophores to those of other optogenetic tools. For cOpn5-expressing cells, merely $10 \mathrm{~ms}$ blue light pulses at the intensity of $16 \mu \mathrm{W} / \mathrm{mm}^{2}$ evoke rapid increase in $\mathrm{Ca}^{2+}$ signals with the peak amplitudes of 3-8 $\Delta F / F$. By contrast, prior characterizations and this study show that the activation of opto-a1AR or mammalian Opn4, the two proposed optogenetic tools for $G_{q}$ signaling, require -3 -order higher light intensity $\left(7-40 \mathrm{~mW} / \mathrm{mm}^{2}\right)$ and prolonged light exposure (20-60 s) but produce only weak $\mathrm{Ca}^{2+}$ signals $(0.25-1 \Delta F / F)$. Therefore, opto-a1AR or mammalian Opn4 cannot mimic the rapid activation profiles of endogenous $\mathrm{G}_{\mathrm{q}}$-coupled receptors that often trigger strong $\mathrm{Ca}^{2+}$ release upon the application of their corresponding ligands. We demonstrate the power of cOpn5 optogenetics by showing the striking physiological and behavioral effects in response to copn5-mediated optical activation of astrocytes and neurons in vivo. By contrast, recent ex vivo studies show that opto-a1AR- and Opn4-mediated optogenetic stimulations slightly increase the amplitudes of $\mathrm{Ca}^{2+}$ signals and only mildly modulate the frequency of $\mathrm{Ca}^{2+}$ transients and synaptic events even after prolonged illumination ${ }^{28,29}$. By overcoming the limitations of light sensitivity, temporal resolution, and response amplitudes associated with opto-a1AR- and Opn4-mediated optogenetics, cOpn5 should find broad applicability for studying $\mathrm{G}_{\mathrm{q}}$ signaling in numerous cells and tissues.

cOpn5 optogenetics also enjoys the benefits of safety and convenience. Although Opn5 from many species are reported UV-responsive ${ }^{36}$, cOpn5 is optimally activated by $470 \mathrm{~nm}$ blue light, which penetrates better than UV and avoids UV-associated cellular toxicity. Its ultra-sensitivity to light minimizes potential heating artifact. It is two-photon activable using long-wavelength light (920 $\mathrm{nm}$ here), suggesting that it is suitable for even deeper tissue activation using a pulsed laser. cOpn5 is strongly, and repetitively activated by light without the 
requirement for exogenous chromophore, possibly because cOpn5 is a bistable opsin that covalently binds to endogenous retinal and is thus resistant to photobleaching ${ }^{32,84}$. By contrast, some studies indicate that the mammalian Opn4 requires the addition of chromophore for continuous activation ${ }^{25,26}$. cOpn5 as a single-component system is particularly useful for both in vitro and in vivo studies as it avoids the burden of delivering a compound into the tissue during the experiment.

cOpn5 optogenetics has several major advantages over chemogenetics and uncaging tools. It is temporally much more precise and offers single-cell or even subcellular spatial resolution. Although $\mathrm{CNO} / \mathrm{hM} 3 \mathrm{Dq}$-mediated chemogenetics has been used to investigate the physiological and behavioral functions of non-excitable cells, such as astrocytes in the brain ${ }^{85,86}$. The diffusive nature of compounds indicates that it is nearly impossible to chemogenetically stimulate $G_{q}$ signaling with cellular and subcellular resolution. cOpn5 also differs from caged compound-based 'uncaging' tools such as caged calcium and caged $\mathrm{IP}_{3}$, since these tools require compound preloading and only partially mimic the $\mathrm{Ca}^{2+}$-related pathways associated with $\mathrm{G}_{\mathrm{q}}$ signaling. There exist other 'uncaging' tools, such as caged glutamate and caged ATP ${ }^{87,88}$, that target endogenous receptors. However, these caged compounds lack cell selectivity and require their introduction into extracellular medium or the intracellular cytoplasm, which limits their applications in behaving animals.

cOpn5 optogenetics should be particularly useful for precisely activating intracellular $\mathrm{G}_{\mathrm{q}}$ signaling, which subsequently triggers $\mathrm{Ca}^{2+}$ release from intracellular stores and activates PKC. cOpn5 differs from current channel-based optogenetic tools, such as ChR2 and its variants, which translocate cations across the plasma membrane. By controlling cellular membrane potentials and thus action potential firing, ChR2 and its variants have contributed tremendously to functional dissection of neural circuits; however, their successes have been more constrained in studying non-excitable cells that lack active ion channels for generating action potentials ${ }^{13}$. In 
addition to the applications on non-excitable cells, cOpn5 optogenetics can also stimulate $\mathrm{G}_{\mathrm{q}}$ signaling in neurons and control animal behavior in a circuit-dependent manner. Of note, $\mathrm{G}_{\mathrm{q}}$-coupled GPCRs may affect variable downstream signaling in a receptor- and cell-specific manner ${ }^{89}$. Indeed, we observed that the same light illumination parameters produce different activation patterns among neurons. We recommend characterizing and confirming the activation profiles, similar to the applications of other optogenetic and chemogenetic tools. cOpn5-mediated optogenetic activation does not generate strictly time-locked action potential firing as precisely as that by ChR2 in neurons. Ion channel-based optogenetic tools would be preferable if temporally precise control of action potential firing is necessary. Nevertheless, cOpn5-mediated asynchronous firing activity may be useful for circuit dissection, since it avoids the potential artefact of massively synchronized neuronal activation.

In addition to the technical advances, our findings also have several functional implications about astrocyte functions in vivo. Although ATP is considered an important gliotransmitter, previous studies have revealed multiple releasing mechanisms that depend on the methods of stimulation (electrical, neurochemical, or mechanical), the presence of extracellular $\mathrm{Ca}^{2+}$, and the exact form of cell and tissue preparations ${ }^{30,90}$. Though it was reported astrocytes activation could evoke an ATP/Adenosine-dependent transient boost ${ }^{28}$, the presence of ATP release is monitored indirectly. It remained controversial whether activating $G_{q}$ signals in astrocytes triggers ATP release, and if so, how this release is expressed in vivo. Here we provide the first demonstration that stimulating $G_{q}$ signaling pathway within astrocytes triggers massive ATP release in the form of ATP flashes. cOpn5 optogenetics thus provides an ideal technique to study the molecular and cellular mechanisms underlying ATP release. In addition to ATP, astrocyte activation leads to the release of other gliotransmitters, such as D-serine, glutamate, and GABA ${ }^{81}$. The gliotransmitters and their metabolites can exert complex modulatory effects on neuronal excitability and synaptic strength $^{88,91}$. It had remained unclear how these various effects are integrated to modulate 
neuronal activity in vivo. Here we reveal that optogenetic activation of copn5-expressing astrocytes leads to an overall excitatory effect on pyramidal neurons in the S1 cortex of mice. This optogenetic approach lays the foundation for dissecting the molecular, cellular, and circuit mechanisms underlying the rich interactions between astrocytes and neurons. Our experiments in vivo have also demonstrated that cOpn5 has compatibility with optogenetic probes and imaging sensors, such as genetically encoded $\mathrm{Ca}^{2+}$ sensors and GPCR-based neurotransmitter sensors $^{57,92-96}$. cOpn5 together with these sensors potentially allows an all-optical approach to activate $G_{q}$ signaling and simultaneously monitor the relevant effects ${ }^{97}$.

In summary, we present cOpn5 as a blue light-sensitive opsin for rapidly, reversibly, and precisely activating $\mathrm{G}_{\mathrm{q}}$ signaling. We also establish cOpn5 as a powerful and easy-to-use optogenetic tool for activating both non-excitable cells and neurons. Given the ubiquitously important roles of $\mathrm{G}_{\mathrm{q}}$-coupled GPCRs, we expect that cOpn5 will find broad applications for studying the mechanisms and functions of $G_{q}$ signaling in all major cell types and tissues. 


\section{Figure legends}

\section{Fig. 1: cOpn5 mediates optical activation of $G_{q}$ signaling in HEK 293T cells.}

a, Schematic diagram of the putative intracellular signaling in response to light-induced cOpn5 activation. PLC: phospholipase $\mathrm{C} ; \quad \mathrm{PIP}_{2}$ : phosphatidylinositol-4,5-bisphosphate; $\mathrm{IP}_{3}$ : inositol-1,4,5-trisphosphate; $\mathrm{IP}_{1}$ : inositol monophosphate; DAG: diacylglycerol; PKC: protein kinase C; YM-254890: a selective $\mathrm{G}_{\mathrm{q}}$ protein inhibitor.

b, The Cy3-counterstained V5-cOpn5 fusion protein (red) was co-localized with the membrane-tagged EGFP-CAAX (green) in HEK 293T cells. DAPI counterstaining (blue) indicates cell nuclei. Scale bar, $10 \mu \mathrm{m}$.

c, Pseudocolor images of the $\mathrm{Ca}^{2+}$ signals before and after blue light stimulation $(10 \mathrm{~s} ; 100$ $\mu \mathrm{W} / \mathrm{mm}^{2} ; 488 \mathrm{~nm}$ ) in HEK 293 T cells expressing Opn5 from three species (Gallus gallus, Homo sapiens, and Mus musculus). Scale bar, $10 \mu \mathrm{m}$.

d, Time courses of light-evoked $\mathrm{Ca}^{2+}$ signals for cells shown in $\mathbf{c}$. Blue lines above the curves indicate light stimulation. $\mathrm{Ca}^{2+}$ indicator fluorescence intensity relative to its resting fluorescence intensity; abbreviated as $\Delta \mathrm{F} / \mathrm{F}$.

e, The $\mathrm{G}_{\mathrm{q}}$ protein inhibitor YM-254890 (10 nM) reversibly blocked cOpn5-mediated, light-induced $\mathrm{Ca}^{2+}$ signals $(\mathrm{n}=28 \mathrm{HEK} 293 \mathrm{~T}$ cells).

f, Group data show that the $G_{q}$ protein inhibitor $Y M-254890$ (10 nM) reversibly blocked cOpn5-mediated, light-induced $\mathrm{Ca}^{2+}$ signals ( $\mathrm{N}=28$ HEK $293 \mathrm{~T}$ cells). ${ }^{\star \star \star \star} \mathrm{P}<0.0001$, one way ANOVA. Error bars indicate S.E.M.. 
g, YM suppressed the $\mathrm{IP}_{1}$ accumulation evoked by continuous light stimulation (3 min; $100 \mu$ W/mm $\left.{ }^{2} ; 470 \mathrm{~nm}\right)$ in cOpn5-expressing HEK 293T cells $\left(\mathrm{N}=4\right.$ replications). ). ${ }^{\star \star *} \mathrm{P}<0.005$, unpaired t tests).

Fig. 2: cOpn5 sensitively mediates optical control of $\mathbf{G}_{q}$ signaling with high temporal and spatial resolution.

a, Schematic diagram of selected wavelengths (365, 395, 470, 515, 561, 590, and $630 \mathrm{~nm})$ and the raw traces of $\mathrm{Ca}^{2+}$ signals (top panel) and the mean amplitudes of $\mathrm{Ca}^{2+}$ signal of cOpn5-expressing HEK 293T cells in response to light stimulation with different wavelengths (2 s; $100 \mu \mathrm{W} / \mathrm{mm}^{2}$; bottom panel). Error bars indicate S.E.M..

b, Time course of $\mathrm{Ca}^{2+}$ signals evoked by cOPN5-mediated optical activation using light pulses of different durations $\left(1,5,10,20\right.$, or $50 \mathrm{~ms} ; 16 \mu \mathrm{W} / \mathrm{mm}^{2} ; 470 \mathrm{~nm}$; $\mathrm{N}=49 \mathrm{HEK} 293 \mathrm{~T}$ cells) (top panel) an the mean response magnitudes to light stimulation of different durations $(1,5,10,20$, or $50 \mathrm{~ms} ; 16 \mu \mathrm{W} / \mathrm{mm}^{2} ; 470 \mathrm{~nm}$ ) (bottom panel). Error bars indicate S.E.M..

c, Time course of cOpn5-mediated $\mathrm{Ca}^{2+}$ signals under different light intensity $(0,4.8,8,16$, or 32 $\mu \mathrm{W} / \mathrm{mm}^{2} ; 10 \mathrm{~ms} ; 470 \mathrm{~nm}$, mean \pm S.E.M.; $\mathrm{N}=10$ cells) (top panel) and the mean response magnitude under different light intensities $(0,4.8,8,16$, or $32 \mu \mathrm{W} / \mathrm{mm} 2)$ at $10 \mathrm{~ms}, 470 \mathrm{~nm}(\mathrm{n}=88$ HEK 293 T cells) (bottom panel). Error bars indicate S.E.M.. For $10 \mathrm{~ms}, 16 \mu \mathrm{W} / \mathrm{mm} 2$ stimulation, time to $10 \%$ peak activation $=1.36 \pm 0.55 \mathrm{~s}$; time to $90 \%$ peak activation $=2.37 \pm 0.87 \mathrm{~s}$; decay time $\mathrm{T}=18.66 \pm 4.98 \mathrm{~s}$. 
d, Images of light-induced (63 ms; $17 \mu \mathrm{W}$; arrow points to the stimulation region) $\mathrm{Ca}^{2+}$ signal propagation from the stimulated HEK 293T cell to surrounding cells. Scale bar, $10 \mu \mathrm{m}$.

e, Pseudocolor images showing the process of $\mathrm{Ca}^{2+}$ signal propagation across time of $\mathbf{d}$ (frame $\mathrm{N} /(\mathrm{N}-1)>1)$. Frame interval was $500 \mathrm{~ms}$ and each frame is counted once.

f, Pseudocolor images of the baseline and peak $\mathrm{Ca}^{2+}$ signals $(\triangle F / F O)$ in opto-a1AR-expressing HEK 293T cells. The medium buffer contains $10 \mu \mathrm{M}$ all-trans-retinal. Scale bar, $30 \mu \mathrm{m}$.

g, The lack of effect by $15 \mathrm{~s}$ light stimulation on $\mathrm{Ca}^{2+}$ signals (left panel) and mild effect of $60 \mathrm{~s}$ light stimulation on the $\mathrm{Ca}^{2+}$ in opto-a1AR-expressing HEK 293T cells (right panel; N = 15 cells). Green bars indicate light stimulations.

h, Pseudocolor images of the baseline and peak $\mathrm{Ca}^{2+}$ signals $(\triangle F / F O)$ in mOPN4L-expressing HEK 293T cells. The medium buffer contains $10 \mu \mathrm{M}$ all-trans-retinal. Scale bar, $30 \mu \mathrm{m}$.

i, The left panel shows that without ATR, brief light pulses (10 ms, $\left.16 \mu \mathrm{W} / \mathrm{mm}^{2}, 470 \mathrm{~nm}\right)$ evoked strong $\mathrm{Ca}^{2+}$ signals in cOpn5-expressing cells (blue line; $\mathrm{N}=10 \mathrm{HEK} 293 \mathrm{~T}$ cells) but had no effect on mOPN4L-expressing cells (black line; $N=12$ HEK 293T cells). The right panel shows the effect of $25 \mathrm{~s}, 40 \mathrm{~mW} / \mathrm{mm}^{2}$ light stimulation on the $\mathrm{Ca}^{2+}$ in mOPN4L-expressing HEK 293T cells within $10 \mu \mathrm{M}$ ATR ( $N=12$ cells; red line) and the lack of such effect following ATR removal (black line).

Fig. 3: copn5-mediated optogenetics activation of neurons and changes of mouse behaviors in a neural circuit-dependent manner. 
a, Schematic diagram shows the experimental setup for optogenetic stimulation and $\mathrm{Ca}^{2+}$ imaging. We used AAV vectors to express cOpn5, EGFP, and the red $\mathrm{Ca}^{2+}$ sensor jRECO1a in neurons.

b, Pseudocolor images show $\mathrm{Ca}^{2+}$ signals before and after light stimulation $\left(10 \mathrm{~s} ; 100 \mu \mathrm{W} / \mathrm{mm}^{2}\right.$; $473 \mathrm{~nm})$. Scale bar, $10 \mu \mathrm{m}$.

c, Group data of $\mathrm{Ca}^{2+}$ signal traces of 6 individual neurons shown in $\mathbf{b}$.

d, Schematic diagram of the experimental setup for cOpn5 optogenetics and food intake assay. cOpn5 and EGFP were expressed in GABAergic neurons within the LH of VGAT-Cre mice. EGFP was expressed as a control.

e, Summary data show the light-induced $(20 \mathrm{~Hz} ; 5 \mathrm{~ms} /$ pulse; $473 \mathrm{~nm}$; $0.75 \mathrm{~mW}$ output from the fiber tip), cOpn5-mediated activation of eating behaviors. ${ }^{\star \star *} \mathrm{P}=0.0003$; n.s., non-significant; $\mathrm{N}=$ 6 mice; unpaired $t$ test. Error bars indicate S.E.M..

f, Schematic diagram of the experimental setup for food foraging behavior. High-fat food pellets were used. cOpn5 and EGFP were expressed in GABAergic neurons within the ZI of VGAT-Cre mice. EGFP was expressed as a control.

g, Summary data show the cOpn5-mediaed food foraging behaviors. Foraging time percentage was calculated upon receiving light stimulation until the mouse found the hidden food. ${ }^{\star \star * \star P}$ $<0.0001 ; \mathrm{N}=6$ mice; unpaired $t$ test. Error bars indicate S.E.M..

h, Raw data illustrate the pattern of cOpn5-mediated optical activation of GABAergic neurons in these two brain areas. 
i, Group data show the neuronal firing rates before and after pulsed $473 \mathrm{~nm}$ light stimulation (1 $\mathrm{Hz}, 5 \mathrm{~s}$; for the $\mathrm{LH}, \mathrm{N}=6$ neurons, ${ }^{\star *} \mathrm{P}=0.0041$, unpaired $\mathrm{t}$ tests; for the $\mathrm{ZI}, \mathrm{N}=5$ neurons, ${ }^{\star \star} \mathrm{P}=$ 0.0027, unpaired t tests).

\section{Fig. 4 cOpn5-mediated optical activation of astrocytes evokes calcium signal.}

a, Pseudocolor images of the baseline and peak $\mathrm{Ca}^{2+}$ signals following light stimulation of cOpn5-expressing astrocytes. Scale bar, $20 \mu \mathrm{m}$.

b. Plot of $\mathrm{Ca}^{2+}$ signals and heat map representation of $\mathrm{Ca}^{2+}$ signals across trials $(\mathrm{n}=25$ astrocytes).

c, cOpn5 was expressed in cultured primary astrocytes using AAV-cOpn5-T2A-EGFP (green). Astrocyte identity was confirmed by GFAP immunostaining (red). Scale bar, $20 \mu \mathrm{m}$.

d, Images of light-induced $\mathrm{Ca}^{2+}$ signal propagation in a single cOpn5-expressing primary astrocyte stimulated in a subcellular region (stimulation size $4 \times 4 \mu \mathrm{m}^{2}$ and frame interval $=300$ ms). Scale bar, $10 \mu \mathrm{m}$.

e, Images of light-induced $\mathrm{Ca}^{2+}$ signal propagation in cOpn5-expressing primary astrocytes. Scale bar, $10 \mu \mathrm{m}$.

f, Pseudocolor images showing the process of $\mathrm{Ca}^{2+}$ signal propagation across time of $\mathbf{g}$ (frame $\mathrm{N} /(\mathrm{N}-1)>1)$. Frame interval was $500 \mathrm{~ms}$ and each frame is counted once.

g, Schematic diagram of the experimental setup for in vivo two-photon imaging (920 $\mathrm{nm})$ of astrocyte $\mathrm{Ca}^{2+}$ imaging following cOpn5-mediated astrocyte activation. Images show the 
expression of GCaMP6s (green) and cOpn5-T2A-mCherry (red) in astrocytes within the mouse

S1 cortex. Scale bar, $100 \mu \mathrm{m}$.

h, Time courses of light-evoked $\mathrm{Ca}^{2+}$ signals in astrocytes $(\mathrm{N}=5)$.

i, Images of the $\mathrm{Ca}^{2+}$ signals before (basal) and 5s after light stimulation (light). Scale bar, 100 $\mu \mathrm{m}$.

j, Raw traces of eight individual GCaMP6s-expressing astrocytes signals in a mouse that expressed cOpn5 or mCherry.

Fig. 5: cOpn5-mediated optogenetic activation of astrocytes induces massive ATP flashes

\section{and neuronal activation in vivo.}

a, Schematic diagram of the experimental setup for in vivo two-photon imaging (920 nm) of ATP signal following cOpn5-mediated optical activation of astrocytes. We infused two AAV vectors to express cOpn5-T2A-mCherry (red), and $\mathrm{GRAB}_{\mathrm{ATP}}$ sensor (green) in the astrocytes within the mouse S1 cortex.

b, Pseudocolor images show that AAV-GfaABC1D-cOpn5-T2A-mCherry drove mCherry expression in S1 cortical cells that were immunopositive to GFAP, a marker of astrocytes. Scale bar, $20 \mu \mathrm{m}$.

c, Out of 320 cOpn5-mCherry+ cells examined, 312 cells were GFAP+, indicating 97.5\% precision. Within the same imaged areas, a total of 409 cells were GFAP, indicating $76.3 \%$ efficiency of astrocyte labeling using the AAV-GFaABC1D constructs. 
d, Representative images show astrocytic ATP flash events in a cOpn5-expressing mouse in the first 4.5min, with different colors indicating individual flashes. Scale bar, $100 \mu \mathrm{m}$.

e, Images show the overall ATP flash events in a control mouse (no cOpn5 expression), a CNO (2 mg/kg) - treated hM3Dq expression mouse (no cOpn5 expression) and a cOpn5-expressing mouse. The left column shows the raw $\mathrm{GRAB}_{\text {ATP }}$ images at the basal level (before light delivery); the middle column shows $\mathrm{GRAB}_{\text {ATP }}$ signals at $5 \mathrm{~min}$; and the right column shows pseudo-color-coded ATP flash events accumulated during 0-20 min. Scale bar, $100 \mu \mathrm{m}$.

f, The plot of astrocytic ATP flash events number per min across time (0-10 min) in control group (no cOpn5 expression), CNO-treated hM3Dq expression group (no cOpn5 expression), and cOpn5-expressing group.

g, The quantification of ATP flash events number in 20min of the control $(N=6), h M 3 D q+C N O$ $(\mathrm{N}=5)$, and cOpn5 groups $(\mathrm{N}=5)$. For the control - hM3Dq+CNO comparison, no significant difference; for the $\mathrm{hM} 3 \mathrm{Dq}+\mathrm{CNO}-\mathrm{cOpn5}$ comparison, ${ }^{\star \star *} \mathrm{P}=0.0009 ;$ for the control - cOpn5 comparison, ${ }^{* \star *} \mathrm{P}=0.0003$, unpaired $t$ tests.

$\mathbf{h}$, Schematic diagram of the experimental setup for in vivo two-photon imaging (920 $\mathrm{nm})$ of neuron $\mathrm{Ca}^{2+}$ imaging following copn5-mediated astrocyte activation. Images show the expression of cOpn5-T2A-mCherry (red) in astrocytes and the expression of a GCaMP7b (green) in neurons within the mouse S1 cortex. Scale bar, $100 \mu \mathrm{m}$.

i, The plot of average spike rate relative to basal across time $(0-20 \mathrm{~min}) . \mathrm{N}=193$ neurons from 3 mice 
j, Decoded spike rate analysis of GCaMP7b-expressing neurons within the periods of 0-5 min and 15-20 min that were coupled with cOpn5-mediated astrocytes activation (left panel) $\mathrm{N}=193$ neurons). ${ }^{* * \star *} \mathrm{P}<0.0001$, unpaired $t$ test. The number of $\mathrm{Ca}^{2+}$ transients of GCaMP7b-expressing neurons during 0-5 min and 15-20 min, coupled with cOpn5-mediated astrocytes activation (right panel). $\mathrm{N}=193$ neurons, ${ }^{\star \star \star *} \mathrm{P}<0.0001$, Unpaired $t$ test.

k, Decoded spike rate analysis of GCaMP7b-expressing neurons within the periods of $0-5$ min and 15-20 min that were coupled with imaging from mice with mCherry expression in astrocytes as control (left panel) $(\mathrm{N}=170$ neurons). no significant difference; unpaired $t$ test. The number of $\mathrm{Ca}^{2+}$ transient analysis of GCaMP7b-expressing neurons during the periods of 0-5 min and 15-20 min in mice that expressed mCherry in astrocytes (right panel). $\mathrm{N}=170$ neurons, ${ }^{*} \mathrm{P}=0.0146$, paired $t$ test. 


\section{Extended Data Figure Legends}

\section{Extended Data Fig. 1 copn5 couples to $G_{q}$ signaling.}

a, Time course of optically evoked $\mathrm{Ca}^{2+}$ signal in extracellular $\mathrm{Ca}^{2+}$ free medium $(10 \mathrm{~ms} ; 16$ $\mu \mathrm{W} / \mathrm{mm}^{2} ; 470 \mathrm{~nm} ; \mathrm{N}=17$ HEK 293T cells).

b, One representative of phosphorylation of MARCKS in cOpn5-expressing HEK 293T cells in the control group (no light stimulation), the light stimulation group, and light+staurosporine (ST) group (ST, a PKC inhibitor; $10 \mu \mathrm{M}$ ) without addition of retinal.

c, The amount of $p$-MARCKS was normalized to the amount of $\alpha$-tubulin in the same fraction. $N=$ 4, ${ }^{\star \star P} \mathrm{P}=0.0096,{ }^{\star \star \star} \mathrm{P}=0.0004 ;$ Tukey's multiple comparisons test.

d, IP ${ }_{1}$ accumulation in human Opn5-expressing HEK 293T cells with or without light stimulation without addition of retinal. $\mathrm{N}=4$, n.s., no significant difference; unpaired $t$ test.

e, Light has no effect on cAMP levels (10 $\mu \mathrm{M}$ forskolin preincubation) in cOpn5-expressing HEK 293T cells without additional retinal in the medium (left panel) $(N=4)$. Right panel shows the effects of photostimulation on cAMP concentrations for HEK 293T cells expressing Opn5s from four different species following $10 \mu \mathrm{M}$ retinal Preincubation $(\mathrm{N}=4)$. n.s., no significant difference; unpaired $t$ test.

Error bars in c, $\mathbf{d}$ and $\mathbf{e}$ indicate S.E.M..

Extended Data Fig. 2 Direct comparisons between the performance of cOpn5 and hM3Dq chemogenetics. 
a Pseudocolor images (upper) and heat map representation of $\mathrm{Ca}^{2+}$ signals evoked by $10 \mathrm{~s}$ optical stimulation (same as the CNO stimulation time) of cOpn5-expressing cells across 5 consecutive trials. Scale bar, $20 \mu \mathrm{m}$.

b, Effect of chemogenetic stimulation on the $\mathrm{Ca}^{2+}$ signals in hM3Dq-expressing HEK 293T cells.

c, Time courses of $\mathrm{Ca}^{2+}$ signals evoked by cOpn5-mediated optogenetic stimulation (10 s) and hM3Dq-mediated chemogenetic stimulation using CNO puff (100 nM; 10 s), respectively $(\mathrm{N}=20$, cOpn5-expressing HEK 293T cells and 20 hM3Dq-expressing HEK 293T cells).

\section{Extended Data Fig. 3 cOpn5-mediated optogenetics reliably activates neurons.}

a, Schematic diagram depicts optogenetic stimulation and whole-cell patch-clamp recording of cOpn5-expressing neurons in the cortex, striatum, hippocampus, lateral hypothalamus (LH), and zonal incerta (ZI).

b, Raw data illustrate two representative patterns of light-evoked neuronal activation. One neuron (left panels) exhibited rapid membrane potential depolarization and large inward currents ( $1 \mathrm{~Hz}, 5 \mathrm{~s}, 10 \mathrm{~ms} /$ pulse), and another neuron exhibited strong, delayed firing of action potentials yet small sustained inward currents in response to the light pulses.

c, Group data show the neuronal firing rates before and after pulsed $473 \mathrm{~nm}$ light stimulation (20 $\mathrm{Hz}, 1 \mathrm{~s} ; \mathrm{N}=29$ neurons; ${ }^{* \star *} \mathrm{P}<0.0001$, unpaired $t$ test). 
d, Raw trace shows that cOpn5 mediated reliable and reproducible photoactivation of a neuron (left). The right panel shows the summary of firing rates across repetitive trials of light stimulation ( $N=3$ neurons).

e, Images show the expression of EGFP control and bicistronic expression of EGFP and cOpn5 in the LH (white dashed lines). Lesion sites and blue dashed lines indicate the placement of optical fibers. Scale bars, $500 \mu \mathrm{m}$.

f, The injection sites and optical fiber placement in the ZI. Scale bars, $500 \mu \mathrm{m}$.

\section{Extended Data Fig. 4 cOpn5-mediated optical activation of astrocytes, produces reliable}

\section{ATP release and the activation of surrounding neurons.}

a, Time courses of light-evoked Ca2+ events in astrocytes for 20min constant stimulation $(\mathrm{N}=5)$.

b, Pseudocolor color image show total ATP flash events (0-20 min) in cOpn5-expressing mouse in a repeat trial 1 hour after the initial trial.

c, Raw traces of ten individual GCaMP7b-expressing neurons signals in 0-5 min and 15-20 min, coupled with cOpn5-mediated optical activation of astrocytes .

d, Raw traces of ten individual GCaMP7b-expressing neurons signals during the periods of 0-5 min and 15-20 min in a mouse that expressed mCherry in astrocytes. 
Supplementary Table 1: Comparison of chicken, human, and mouse Opn5.

Supplementary Table 2: Key resources.

Supplementary Table 3: Summary of statistical analyses.

Supplementary Table 4: Comparison cOpn5 with other optogenetic and chemogenetic tools.

Video 1. cOpn5-mediated optical activation of $\mathrm{Ca}^{2+}$ signals in HEK 293T cells (left) and astrocytes (right). Related to Figure 1 and Figure 4. Caption indicated the period of the light on. Scale bars, $500 \mu \mathrm{m}$.

Video 2. Subcellular stimulation and $\mathrm{Ca}^{2+}$ waves in HEK 293 cells (left) and in astrocytes (right). Related to Figure 2 and Figure 4. Caption indicated the restricting brief light stimulation (63 ms) and the blue dotted arrows indicated the stimulation sites.

Video 3. cOpn5-mediated optogenetics changes mouse behaviors. The video first shows the behavior effect of optogenetically activating ZI GABAergic neurons and then the behavior effect of activating LH GABAergic neurons. Related to Figure 3.

Video 4. copn5-mediated optical activation of $\mathrm{Ca}^{2+}$ signals in a control mouse and cOpn5-expressing mouse with blue light stimulation. Related to Figure 4.

Video 5. ATP flash events in a control mouse (no cOpn5 expression), a mouse 40min after CNO treatment (hM3Dq expression), a copn5-expressing mouse during the initial imaging period (lower left) and during the second imaging session (lower right). Related to Figure 5. 


\section{References}

1 Hauser, A. S., Attwood, M. M., Rask-Andersen, M., Schioth, H. B. \& Gloriam, D. E. Trends in GPCR drug discovery: new agents, targets and indications. Nat Rev Drug Discov 16, 829-842, doi:10.1038/nrd. 2017. 178 (2017).

2 Wettschureck, N. \& Offermanns, S. Mammalian G proteins and their cell type specific functions. Physiol Rev 85, 1159-1204, doi:10. 1152/physrev. 00003. 2005 (2005).

3 Exton, J. H. Regulation of phosphoinositide phospholipases by hormones, neurotransmitters, and other agonists linked to G proteins. Annu Rev Pharmacol Toxicol 36, 481-509, doi:10.1146/annurev. pa. 36. 040196. 002405 (1996).

4 Ritter, S. L. \& Hall, R. A. Fine-tuning of GPCR activity by receptor-interacting proteins. Nature reviews Molecular cell biology 10, 819-830 (2009).

5 Kadamur, G. \& Ross, E. M. Mammalian phospholipase C. Annu Rev Physiol 75, 127-154, doi:10. 1146/annurev-physiol-030212-183750 (2013).

6 Clapham, D. E. Calcium signaling. Cell 131, 1047-1058 (2007).

7 Boyden, E. S., Zhang, F., Bamberg, E., Nage1, G. \& Deisseroth, K. Millisecond-timescale, genetically targeted optical control of neural activity. Nat Neurosci 8, 1263-1268, doi:10.1038/nn1525 (2005).

8 Fenno, L., Yizhar, 0. \& Deisseroth, K. The Development and Application of Optogenetics. Annual Review of Neuroscience 34, 389-412,

doi : 10. 1146/annurev-neuro-061010-113817 (2011).

9 Quadrato, G. et al. Cell diversity and network dynamics in photosensitive human brain organoids. Nature 545, 48-53 (2017).

10 Rost, B. R., Schneider-Warme, F., Schmitz, D. \& Hegemann, P. Optogenetic tools for subcellular applications in neuroscience. Neuron 96, 572-603 (2017).

11 Tye, K. M. \& Deisseroth, K. Optogenetic investigation of neural circuits underlying brain disease in animal models. Nature Reviews Neuroscience 13, 251-266 (2012).

12 Zhang, F. et al. The microbial opsin family of optogenetic tools. Cell 147, 1446-1457 (2011).

13 Adams, S. R. \& Tsien, R. Y. Controlling cell chemistry with caged compounds. Annual review of physiology 55, 755-784 (1993).

14 Gomez, J. L. et al. Chemogenetics revealed: DREADD occupancy and activation via converted clozapine. Science 357, 503-507 (2017).

15 Herlitze, S. \& Landmesser, L. T. New optical tools for controlling neuronal activity. Current opinion in neurobiology 17, 87-94 (2007). 
16 Spoida, K. et al. Melanopsin variants as intrinsic optogenetic on and off switches for transient versus sustained activation of $G$ protein pathways. Current Biology 26, 1206-1212 (2016).

17 Spoida, K., Masseck, 0. A., Deneris, E. S. \& Herlitze, S. Gq/5-HT2c receptor signals activate a local GABAergic inhibitory feedback circuit to modulate serotonergic firing and anxiety in mice. Proceedings of the National Academy of Sciences 111, 6479-6484 (2014).

18 Vaezy, S. et al. Real-time visualization of high-intensity focused ultrasound treatment using ultrasound imaging. Ultrasound in medicine \& biology 27, 33-42 (2001).

19 Airan, R. D., Thompson, K. R., Fenno, L. E., Bernstein, H. \& Deisseroth, K. Temporally precise in vivo control of intracellular signalling. Nature 458, 1025-1029, doi:10. 1038/nature07926 (2009).

20 Güler, A. D. et al. Melanopsin cells are the principal conduits for rodcone input to non-image-forming vision. Nature 453, 102-105 (2008).

21 Hankins, M. W., Peirson, S. N. \& Foster, R. G. Melanopsin: an exciting photopigment. Trends in neurosciences 31, 27-36 (2008).

22 Hattar, S., Liao, H. -W., Takao, M., Berson, D. M. \& Yau, K. -W. Melanopsin-containing retinal ganglion cells: architecture, projections, and intrinsic photosensitivity. Science 295, 1065-1070 (2002).

23 Xue, T. et al. Melanopsin signalling in mammalian iris and retina. Nature 479, 67-73 (2011).

24 Panda, S. et al. Melanopsin is required for non-image-forming photic responses in blind mice. Science 301, 525-527 (2003).

25 Melyan, Z., Tarttelin, E. E., Bellingham, J., Lucas, R. J. \& Hankins, M. W. Addition of human melanopsin renders mammalian cells photoresponsive. Nature 433, 741-745, doi:10. 1038/nature03344 (2005).

26 Qiu, X. et al. Induction of photosensitivity by heterologous expression of melanopsin. Nature 433, 745-749 (2005).

27 Mure, L. S. et al. Melanopsin-encoded response properties of intrinsically photosensitive retinal ganglion cells. Neuron 90, 1016-1027 (2016).

28 Mederos, S. et al. Melanopsin for precise optogenetic activation of astrocyte - neuron networks. Glia 67, 915-934 (2019).

29 Gerasimov, E. et al. Optogenetic Activation of Astrocytes-Effects on Neuronal Network Function. International Journal of Molecular Sciences 22, 9613 (2021).

30 Figueiredo, M. et al. Comparative analysis of optogenetic actuators in cultured astrocytes. Cell calcium 56, 208-214 (2014).

31 Terakita, A. The opsins. Genome biology 6, 1-9 (2005).

32 Koyanagi, M. \& Terakita, A. Diversity of animal opsin-based pigments and 
their optogenetic potential. Biochim Biophys Acta 1837, 710-716, doi:10. 1016/j. bbabio. 2013. 09. 003 (2014).

33 Yau, K. -W. \& Hardie, R. C. Phototransduction motifs and variations. Cell 139, 246-264 (2009).

34 Copits, B. A. et al. A photoswitchable GPCR-based opsin for presynaptic inhibition. Neuron 109, 1791-1809 e1711, doi:10. 1016/ j. neuron. 2021. 04. 026 (2021).

35 Mahn, M. et al. Efficient optogenetic silencing of neurotransmitter release with a mosquito rhodopsin. Neuron 109, 1621-1635 e1628,

doi:10. 1016/j. neuron. 2021.03. 013 (2021).

36 Kojima, D. et al. UV-sensitive photoreceptor protein OPN5 in humans and mice. PLoS One 6, e26388, doi:10.1371/journal. pone. 0026388 (2011).

37 Yamashita, T. et al. Opn5 is a UV-sensitive bistable pigment that couples with Gi subtype of G protein. Proc Nat1 Acad Sci U S A 107, 22084-22089, doi:10. 1073/pnas. 1012498107 (2010).

38 Zhang, K. X. et al. Violet-light suppression of thermogenesis by opsin 5 hypothalamic neurons. Nature 585, 420-425, doi:10.1038/s41586-020-2683-0 (2020).

39 Calligaro, H., Dkhissi-Benyahya, 0. \& Panda, S. Ocular and extraocular roles of neuropsin in vertebrates. Trends in neurosciences (2021).

40 Rios, M. N., Marchese, N. A. \& Guido, M. E. Expression of Non-visual Opsins Opn3 and Opn5 in the Developing Inner Retinal Ce11s of Birds. Light-Responses in Muller Glial Cells. Front Cell Neurosci 13, 376,

doi:10. 3389/fnce1. 2019. 00376 (2019).

41 Nakane, Y. et al. A mammalian neural tissue opsin (Opsin 5) is a deep brain photoreceptor in birds. Proceedings of the National Academy of Sciences 107, 15264-15268 (2010).

42 Nakane, Y., Shimmura, T., Abe, H. \& Yoshimura, T. Intrinsic photosensitivity of a deep brain photoreceptor. Current Biology 24, R596-R597 (2014).

43 Brueggemann, L. I. \& Sullivan, J. M. HEK293S cells have functional retinoid processing machinery. The Journal of general physiology 119, 593-612 (2002).

44 Taniguchi, M. et al. Structure of YM-254890, a Nove1 Gq/11 Inhibitor from Chromobacterium sp. QS3666. Tetrahedron 59, 4533-4538,

doi:10. 1016/s0040-4020(03) 00680-x (2003).

45 Hartwig, J. et al. MARCKS is an actin filament crosslinking protein regulated by protein kinase C and calcium-calmodulin. Nature 356, 618-622 (1992).

46 Zhang, F., Wang, L. -P. , Boyden, E. S. \& Deisseroth, K. Channelrhodopsin-2 and optical control of excitable cells. Nature methods 3, 785-792 (2006).

47 Lin, J. Y. A user's guide to channelrhodopsin variants: features, limitations and future developments. Experimental physiology 96, 19-25 (2011). 
48 Urban, D. J. \& Roth, B. L. DREADDs (designer receptors exclusively activated by designer drugs): chemogenetic tools with therapeutic utility. Annual review of pharmacology and toxicology 55, 399-417 (2015).

49 Roth, B. L. DREADDs for neuroscientists. Neuron 89, 683-694 (2016).

50 Krashes, M. J. et al. Rapid, reversible activation of AgRP neurons drives feeding behavior in mice. The Journal of clinical investigation 121, 1424-1428 (2011).

51 Rogan, S. C., Roth, B. L. \& Morrow, A. L. Remote Control of Neuronal Signaling. Pharmacological Reviews 63, 291-315, doi:10.1124/pr.110.003020 (2011).

52 Panda, S. et al. Illumination of the melanopsin signaling pathway. Science 307, 600-604 (2005).

53 Jennings, J. H., Rizzi, G., Stamatakis, A. M., Ung, R. L. \& Stuber, G. D. The inhibitory circuit architecture of the lateral hypothalamus orchestrates feeding. Science 341, 1517-1521 (2013).

54 Stuber, G. D. \& Wise, R. A. Lateral hypothalamic circuits for feeding and reward. Nat Neurosci 19, 198-205, doi:10. 1038/nn. 4220 (2016).

55 Li, Y. et al. Hypothalamic Circuits for Predation and Evasion. Neuron 97, 911-924 e915, doi:10. 1016/j. neuron. 2018.01.005 (2018).

56 Zhang, X. \& van den Pol, A. N. Rapid binge-like eating and body weight gain driven by zona incerta GABA neuron activation. Science 356, 853-859, doi:10. 1126/science. aam7100 (2017).

57 Tian, L. et al. Imaging neural activity in worms, flies and mice with improved GCaMP calcium indicators. Nature methods 6, 875-881 (2009).

58 Charles, A. C., Merri11, J. E., Dirksen, E. R. \& Sandersont, M. J. Intercellular signaling in glial cells: calcium waves and oscillations in response to mechanical stimulation and glutamate. Neuron 6, 983-992 (1991).

59 Corne11-Be11, A. H., Finkbeiner, S. M., Cooper, M. S. \& Smith, S. J. Glutamate induces calcium waves in cultured astrocytes: long-range glial signaling. Science 247, 470-473 (1990).

60 Bazargani, N. \& Attwe11, D. Astrocyte calcium signaling: the third wave. Nature neuroscience 19, 182-189 (2016).

61 Agulhon, C. et al. What is the role of astrocyte calcium in neurophysiology? Neuron 59, 932-946 (2008).

62 Lawlor, P. A., Bland, R. J., Mouravlev, A., Young, D. \& During, M. J. Efficient gene delivery and selective transduction of glial cells in the mammalian brain by AAV serotypes isolated from nonhuman primates. Molecular therapy 17, 1692-1702 (2009).

63 Lee, Y., Messing, A., Su, M. \& Brenner, M. GFAP promoter elements required for region - specific and astrocyte - specific expression. Glia 56, 481-493 (2008). 
64 Zonta, M. et al. Neuron-to-astrocyte signaling is central to the dynamic control of brain microcirculation. Nature neuroscience 6, 43-50 (2003).

65 Sofroniew, M. V. Astrocyte barriers to neurotoxic inflammation. Nature Reviews Neuroscience 16, 249-263 (2015).

66 Abbott, N. J., Rönnbäck, L. \& Hansson, E. Astrocyte - endothelial interactions at the blood-brain barrier. Nature reviews neuroscience 7 , 41-53 (2006).

67 Allen, N. J. \& Eroglu, C. Cell biology of astrocyte-synapse interactions. Neuron 96, 697-708 (2017).

68 Linnerbauer, M., Wheeler, M. A. \& Quintana, F. J. Astrocyte crosstalk in CNS inflammation. Neuron (2020).

69 Xie, A. X., Petravicz, J. \& McCarthy, K. D. Molecular approaches for manipulating astrocytic signaling in vivo. Frontiers in cellular neuroscience 9, 144 (2015).

70 Yu, X., Nagai, J. \& Khakh, B. S. Improved tools to study astrocytes. Nature Reviews Neuroscience 21, 121-138 (2020).

71 Davalos, D. et al. ATP mediates rapid microglial response to local brain injury in vivo. Nature neuroscience 8, 752-758 (2005).

72 Zhang, J. - m. et al. ATP released by astrocytes mediates glutamatergic activity-dependent heterosynaptic suppression. Neuron 40, 971-982 (2003).

73 Zhang, Z. et al. Regulated ATP release from astrocytes through lysosome exocytosis. Nature cell biology 9, 945-953 (2007).

74 Stout, C. E., Costantin, J. L., Naus, C. C. \& Charles, A. C. Intercellular calcium signaling in astrocytes via ATP release through connexin hemichannels. Journal of Biological Chemistry 277, 10482-10488 (2002).

75 Coco, S. et al. Storage and release of ATP from astrocytes in culture. Journal of Biological Chemistry 278, 1354-1362 (2003).

$76 \mathrm{Wu}, \mathrm{Z}$. \& Li, Y. New frontiers in probing the dynamics of purinergic transmitters in vivo. Neuroscience research 152, 35-43 (2020).

77 Wu, Z. et al., doi:10. 1101/2021. 02.24.432680 (2021).

78 Adamsky, A. et al. Astrocytic activation generates de novo neuronal potentiation and memory enhancement. Cell 174, 59-71. e14 (2018).

79 Cao, X. et al. Astrocyte-derived ATP modulates depressive-1ike behaviors. Nature medicine 19, 773-777 (2013).

80 Kofuji, P. \& Araque, A. Astrocytes and behavior. Annual Review of Neuroscience 44 (2021).

81 Araque, A. et al. Gliotransmitters travel in time and space. Neuron 81, 728-739 (2014).

82 Butt, A. M. in Seminars in cell \& developmental biology. 205-213 (Elsevier).

83 Kofuji, P. \& Araque, A. G-protein-coupled receptors in astrocyte-neuron 
communication. Neuroscience 456, 71-84 (2021).

84 Tsukamoto, H. \& Terakita, A. Diversity and functional properties of bistable pigments. Photochemical \& Photobiological Sciences 9, 1435-1443 (2010).

85 Agulhon, C. et al. Modulation of the autonomic nervous system and behaviour by acute glial cell Gq protein - coupled receptor activation in vivo. The Journal of physiology 591, 5599-5609 (2013).

86 Shen, W. et al. Chemogenetic manipulation of astrocytic activity: Is it possible to reveal the roles of astrocytes? Biochemical Pharmacology 186, 114457 (2021).

87 Ellis-Davies, G. C. Caged compounds: photorelease technology for control of cellular chemistry and physiology. Nature methods 4, 619-628 (2007).

88 Lezmy, J. et al. Astrocyte Ca $(2+)$-evoked ATP release regulates myelinated axon excitability and conduction speed. Science 374, eabh2858,

doi:10. 1126/science. abh2858 (2021).

89 Rosenbaum, D. M., Rasmussen, S. G. \& Kobilka, B. K. The structure and function of G-protein-coupled receptors. Nature 459, 356-363 (2009).

90 Hamilton, N. B. \& Attwe11, D. Do astrocytes really exocytose neurotransmitters? Nature Reviews Neuroscience 11, 227-238 (2010).

91 Haydon, P. G. GLIA: 1istening and talking to the synapse. Nature Reviews Neuroscience 2, 185-193 (2001).

92 Chen, T. -W. et al. Ultrasensitive fluorescent proteins for imaging neuronal activity. Nature 499, 295-300 (2013).

93 Jing, M. et al. An optimized acetylcholine sensor for monitoring in vivo cholinergic activity. Nature methods 17, 1139-1146 (2020).

94 Sun, F. et al. A genetically encoded fluorescent sensor enables rapid and specific detection of dopamine in flies, fish, and mice. Cell 174, 481-496. e419 (2018).

95 Wan, J. et al. A genetically encoded sensor for measuring serotonin dynamics. Nature Neuroscience 24, 746-752 (2021).

96 Zhao, Y. et al. An expanded palette of genetically encoded Ca2+ indicators. Science 333, 1888-1891 (2011).

97 Hochbaum, D. R. et al. Al1-optical electrophysiology in mammalian neurons using engineered microbial rhodopsins. Nat Methods 11, 825-833, doi:10. 1038/nmeth. 3000 (2014). 


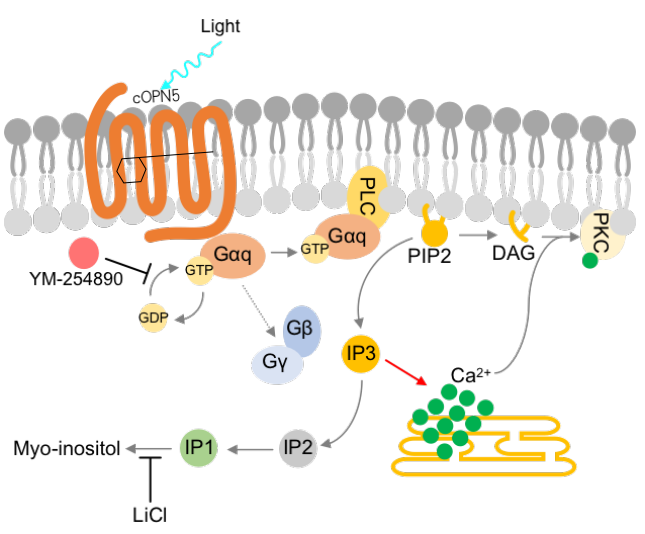

b

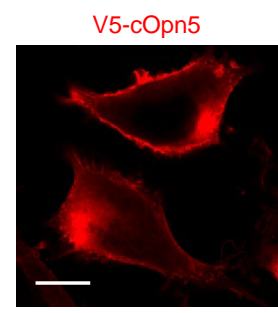

DAPI

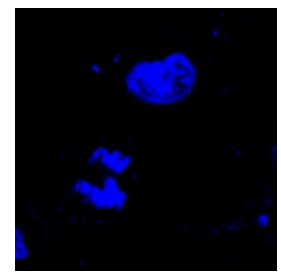

EGFP-CAAX

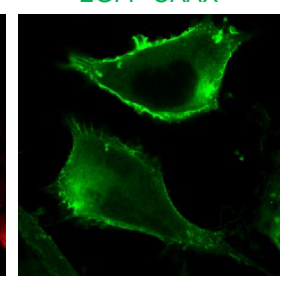

overlay

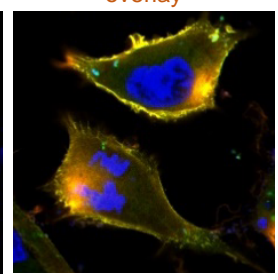

e
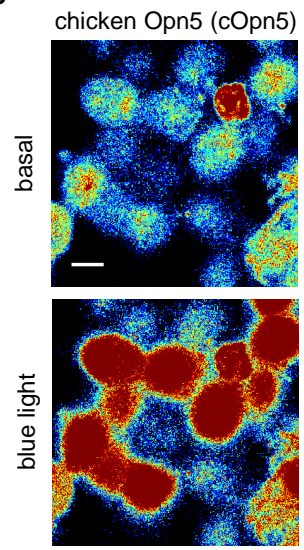

d
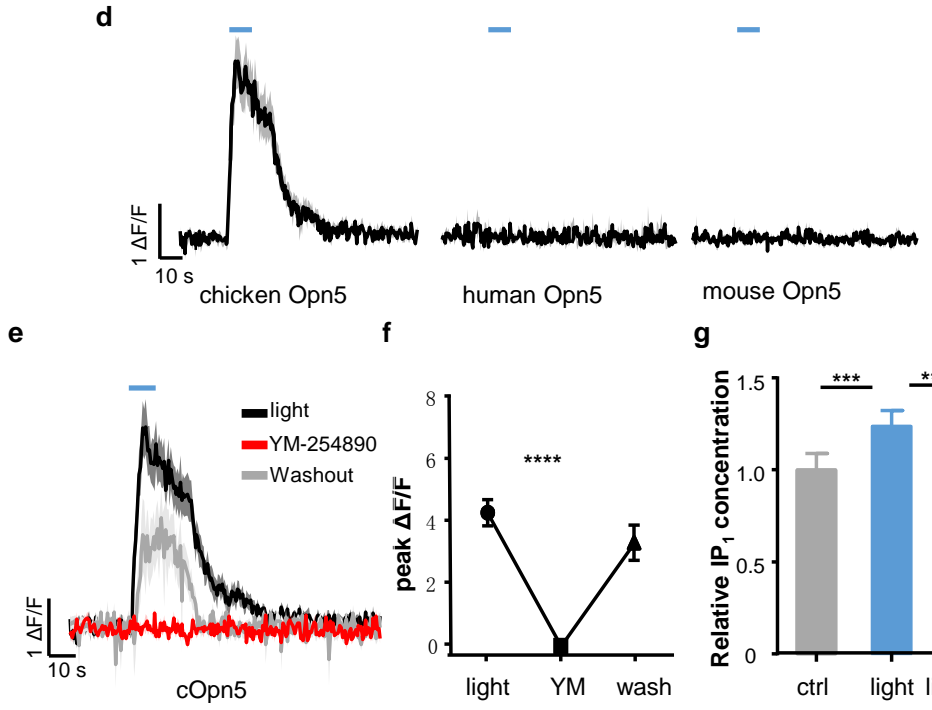
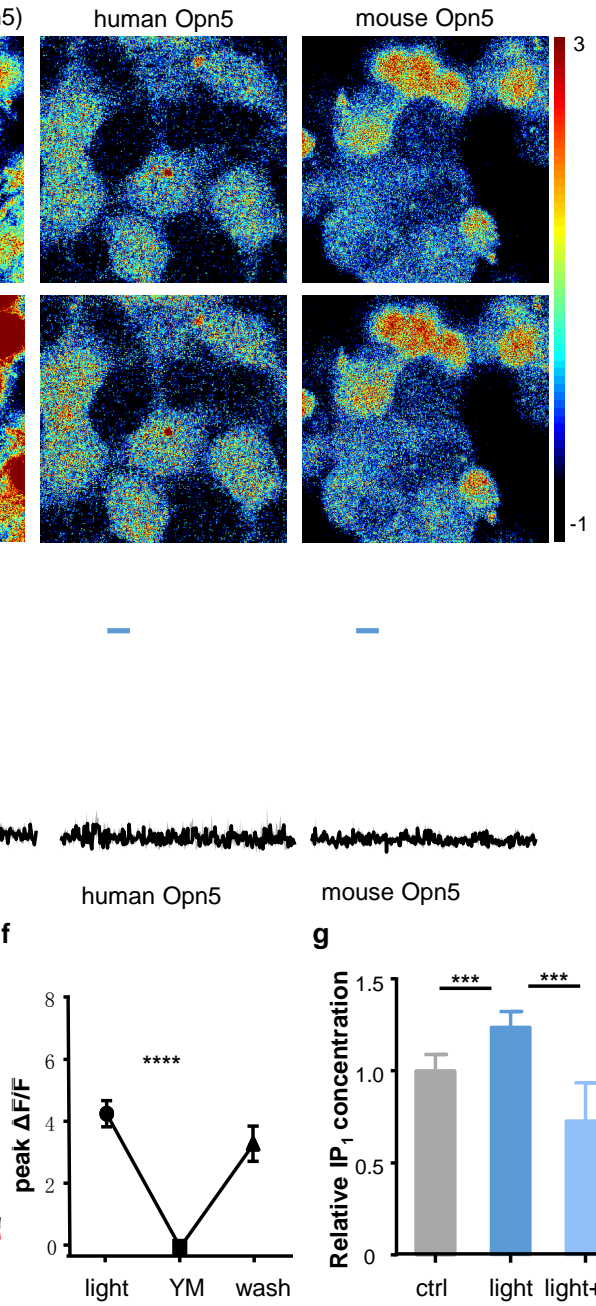

g

mouse Opn5
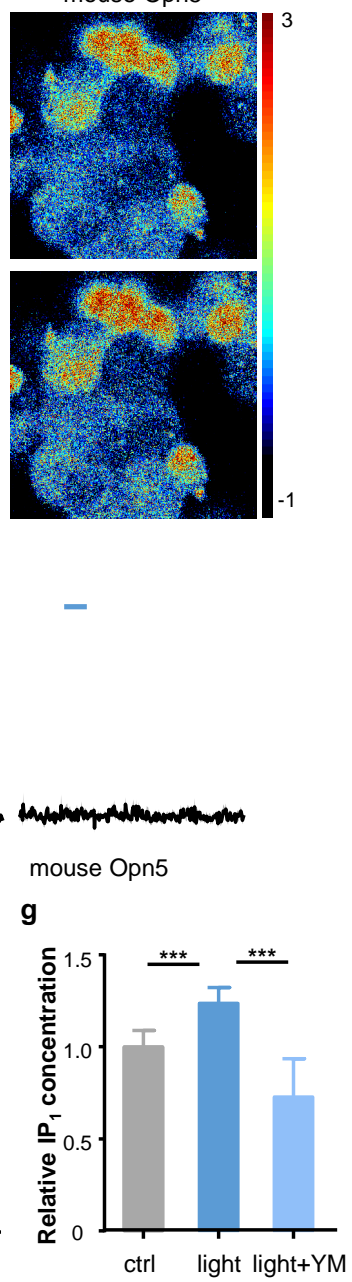

Fig. 1: cOpn5 mediates optical activation of $G_{q}$ signaling in HEK 293T cells.

a, Schematic diagram of the putative intracellular signaling in response to light-induced cOpn5 activation. PLC: phospholipase C; $\mathrm{PIP}_{2}$ : phosphatidylinositol-4,5-bisphosphate; $\mathrm{IP}_{3}$ : inositol-1,4,5trisphosphate; $\mathrm{IP}_{1}$ : inositol monophosphate; DAG: diacylglycerol; PKC: protein kinase C; YM254890: a selective $\mathrm{G}_{\mathrm{q}}$ protein inhibitor.

b, The Cy3-counterstained V5-cOpn5 fusion protein (red) was co-localized with the membranetagged EGFP-CAAX (green) in HEK 293T cells. DAPI counterstaining (blue) indicates cell nuclei. Scale bar, $10 \mu \mathrm{m}$.

c, Pseudocolor images of the $\mathrm{Ca}^{2+}$ signals before and after blue light stimulation (10 s; 100 $\mu \mathrm{W} / \mathrm{mm}^{2} ; 488 \mathrm{~nm}$ ) in HEK 293T cells expressing Opn5 from three species (Gallus gallus, Homo sapiens, and Mus musculus). Scale bar, $10 \mu \mathrm{m}$.

d, Time courses of light-evoked $\mathrm{Ca}^{2+}$ signals for cells shown in c. Blue lines above the curves indicate light stimulation. $\mathrm{Ca}^{2+}$ indicator fluorescence intensity relative to its resting fluorescence intensity; abbreviated as $\Delta F / F$.

e, The $\mathrm{G}_{\mathrm{q}}$ protein inhibitor $\mathrm{YM}-254890(10 \mathrm{nM})$ reversibly blocked cOpn5-mediated, light-induced $\mathrm{Ca}^{2+}$ signals ( $n=28$ HEK 293T cells).

f, Group data show that the $\mathrm{G}_{q}$ protein inhibitor $\mathrm{YM}-254890(10 \mathrm{nM})$ reversibly blocked cOpn5mediated, light-induced $\mathrm{Ca}^{2+}$ signals ( $\mathrm{N}=28 \mathrm{HEK} 293 \mathrm{~T}$ cells). ${ }^{* \star *} \mathrm{P}<0.0001$, one way ANOVA. Error bars indicate S.E.M..

g, YM suppressed the $\mathrm{IP}_{1}$ accumulation evoked by continuous light stimulation (3 min; 100 $\mu \mathrm{W} / \mathrm{mm}^{2} ; 470 \mathrm{~nm}$ ) in cOpn5-expressing HEK 293T cells $\left(\mathrm{N}=4\right.$ replications). ${ }^{\star \star \star} \mathrm{P}<0.005$, unpaired tests). 
(which was not certified by peer review) is the author/funder, who has granted bioRxiv a license to display the preprint in perpetuity. It is $m$ available under aCC-BY-NC-ND 4.0 International license.

a

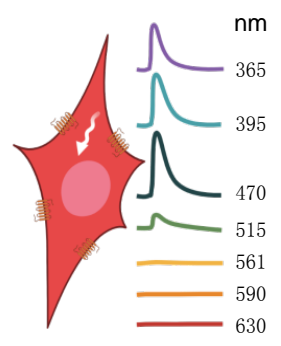

d
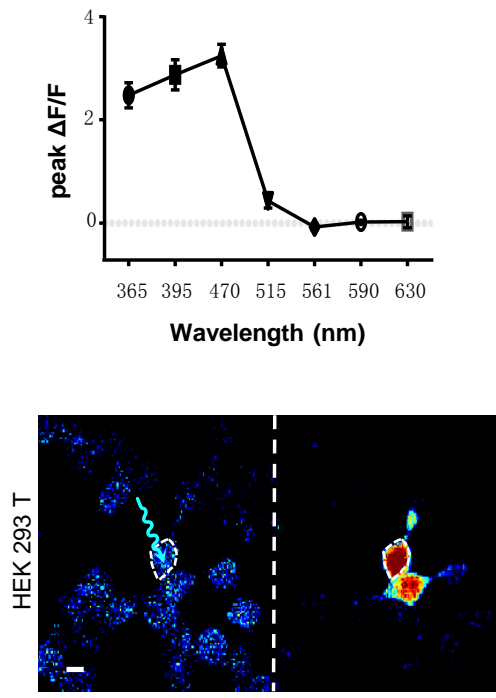

$0 \mathrm{~s}$

basal
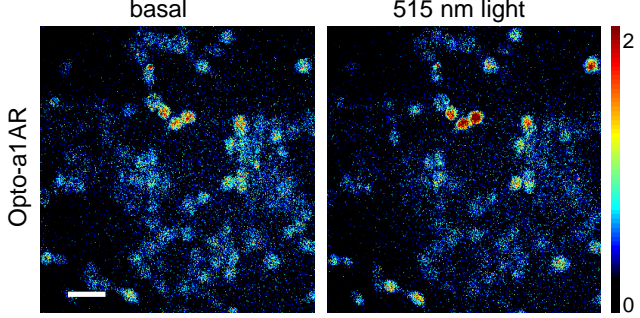

b
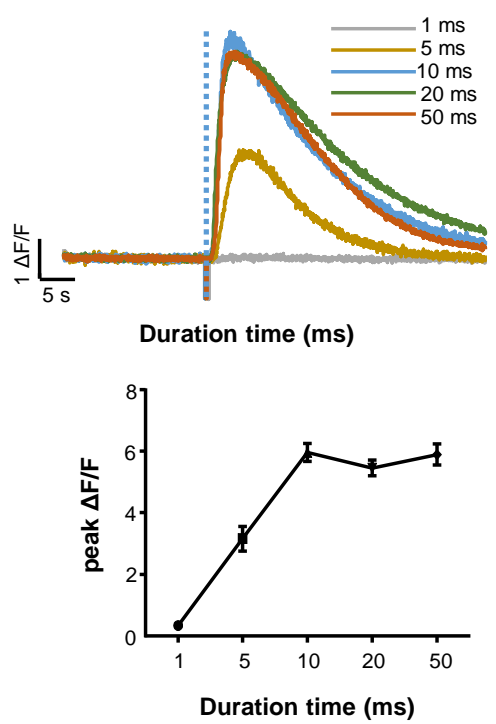

c

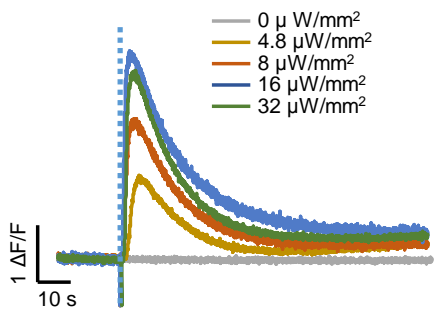

Intensity $\left(\mu \mathrm{W} / \mathrm{mm}^{2}\right)$ mapping

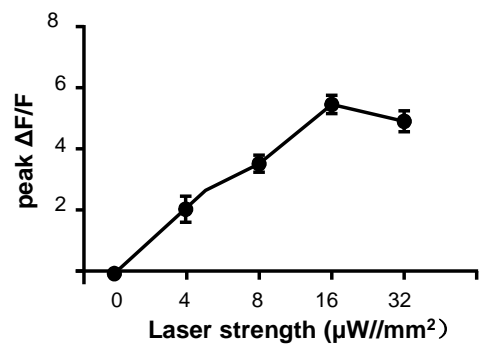

e

h
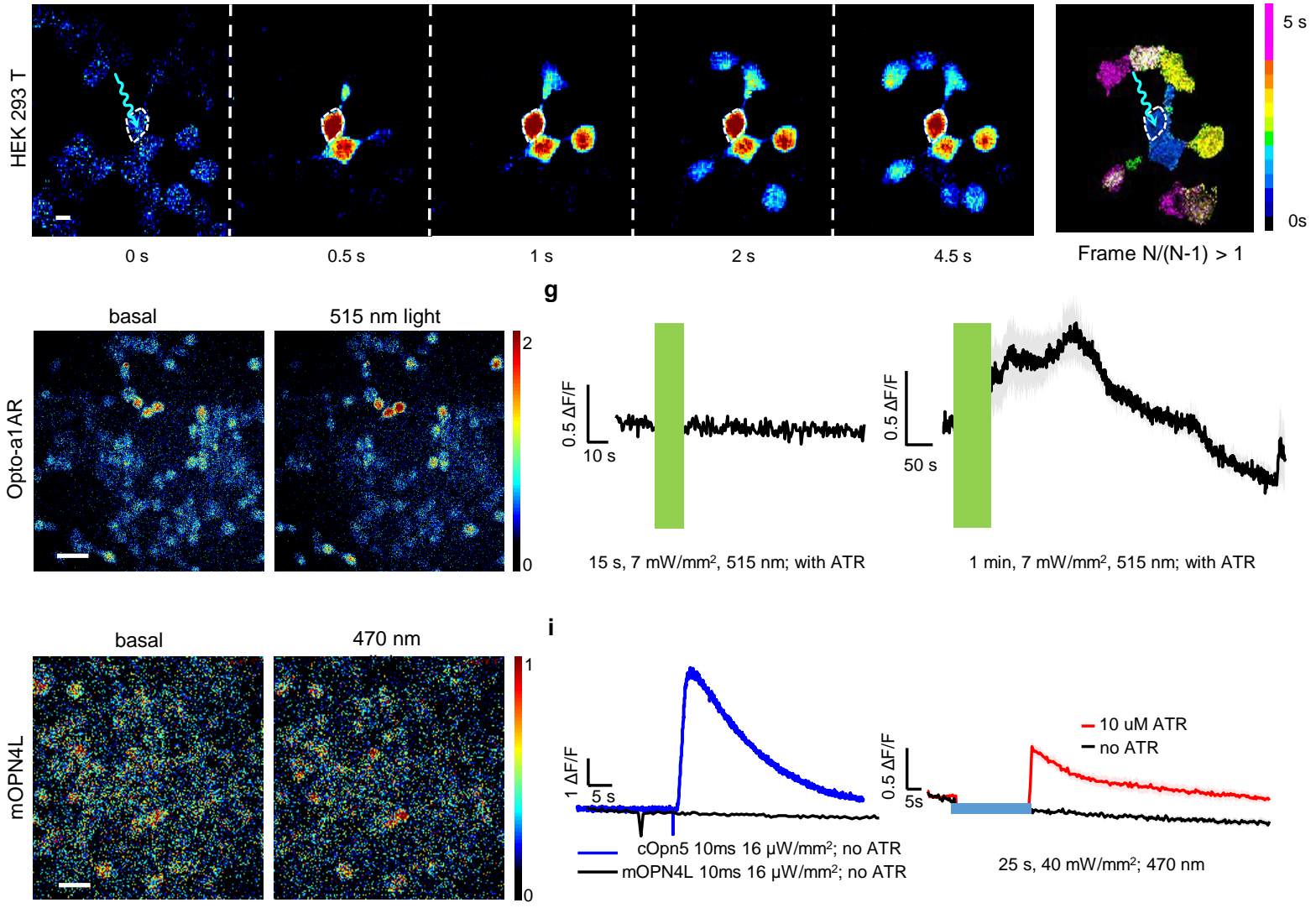

g
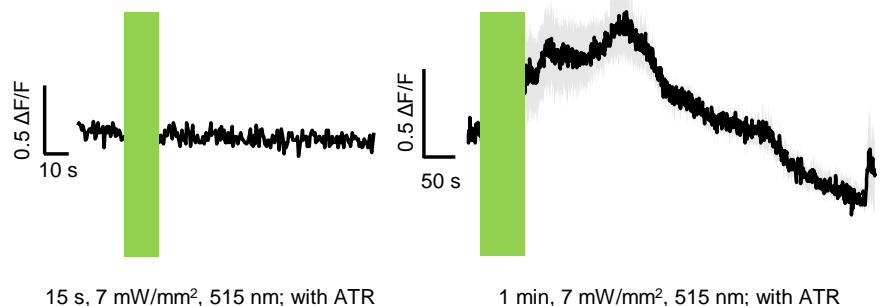

$15 \mathrm{~s}, 7 \mathrm{~mW} / \mathrm{mm}^{2}, 515 \mathrm{~nm}$; with ATR

$1 \mathrm{~min}, 7 \mathrm{~mW} / \mathrm{mm}^{2}, 515 \mathrm{~nm}$; with ATR 


\section{Fig. 2: cOpn5 sensitively mediates optical control of $\mathbf{G}_{\mathrm{q}}$ signaling with high temporal and spatial resolution.}

a, Schematic diagram of selected wavelengths (365, 395, 470, 515, 561, 590, and $630 \mathrm{~nm})$ and the raw traces of $\mathrm{Ca}^{2+}$ signals (top panel) and the mean amplitudes of $\mathrm{Ca}^{2+}$ signal of cOpn5-expressing HEK 293T cells in response to light stimulation with different wavelengths (2 s; $100 \mu \mathrm{W} / \mathrm{mm}^{2}$; bottom panel). Error bars indicate S.E.M..

b, Time course of $\mathrm{Ca}^{2+}$ signals evoked by cOPN5-mediated optical activation using light pulses of different durations $\left(1,5,10,20\right.$, or $50 \mathrm{~ms} ; 16 \mu \mathrm{W} / \mathrm{mm}^{2} ; 470 \mathrm{~nm}$; $\mathrm{N}=49 \mathrm{HEK}$ 293T cells) (top panel) an the mean response magnitudes to light stimulation of different durations $(1,5,10,20$, or 50 ms; $16 \mu \mathrm{W} / \mathrm{mm}^{2} ; 470 \mathrm{~nm}$ ) (bottom panel). Error bars indicate S.E.M..

c, Time course of cOpn5-mediated $\mathrm{Ca}^{2+}$ signals under different light intensity $(0,4.8,8,16$, or 32 $\mu \mathrm{W} / \mathrm{mm}^{2} ; 10 \mathrm{~ms} ; 470 \mathrm{~nm}$, mean \pm S.E.M.; $\mathrm{N}=10$ cells) (top panel) and the mean response magnitude under different light intensities $(0,4.8,8,16$, or $32 \mu \mathrm{W} / \mathrm{mm} 2)$ at $10 \mathrm{~ms}, 470 \mathrm{~nm}(\mathrm{n}=88$ HEK 293T cells) (bottom panel). Error bars indicate S.E.M.. For $10 \mathrm{~ms}, 16 \mu \mathrm{W} / \mathrm{mm} 2$ stimulation, time to $10 \%$ peak activation $=1.36 \pm 0.55 \mathrm{~s}$; time to $90 \%$ peak activation $=2.37 \pm 0.87 \mathrm{~s}$; decay time $\mathrm{\tau}=$ $18.66 \pm 4.98 \mathrm{~s}$.

d, Images of light-induced (63 ms; $17 \mu \mathrm{W}$; arrow points to the stimulation region) $\mathrm{Ca}^{2+}$ signal propagation from the stimulated HEK 293T cell to surrounding cells. Scale bar, $10 \mu \mathrm{m}$.

e, Pseudocolor images showing the process of $\mathrm{Ca}^{2+}$ signal propagation across time of $\mathbf{d}$ (frame $\mathrm{N} /(\mathrm{N}-1)>1)$. Frame interval was $500 \mathrm{~ms}$ and each frame is counted once.

f, Pseudocolor images of the baseline and peak $\mathrm{Ca}^{2+}$ signals $(\triangle F / F O)$ in opto-a1AR-expressing HEK 293T cells. The medium buffer contains $10 \mu \mathrm{M}$ all-trans-retinal. Scale bar, $30 \mu \mathrm{m}$.

$\mathbf{g}$, The lack of effect by $15 \mathrm{~s}$ light stimulation on $\mathrm{Ca}^{2+}$ signals (left panel) and mild effect of $60 \mathrm{~s}$ light stimulation on the $\mathrm{Ca}^{2+}$ in opto-a1AR-expressing HEK 293T cells (right panel; $\mathrm{N}=15$ cells). Green bars indicate light stimulations.

h, Pseudocolor images of the baseline and peak $\mathrm{Ca}^{2+}$ signals $(\triangle F / F O)$ in mOPN4L-expressing HEK 293T cells. The medium buffer contains $10 \mu \mathrm{M}$ all-trans-retinal. Scale bar, $30 \mu \mathrm{m}$.

i, The left panel shows that without ATR, brief light pulses $\left(10 \mathrm{~ms}, 16 \mu \mathrm{W} / \mathrm{mm}^{2}, 470 \mathrm{~nm}\right)$ evoked strong $\mathrm{Ca}^{2+}$ signals in cOpn5-expressing cells (blue line; $\mathrm{N}=10 \mathrm{HEK} 293 \mathrm{~T}$ cells) but had no effect on mOPN4Lexpressing cells (black line; $\mathrm{N}=12$ HEK 293T cells). The right panel shows the effect of $25 \mathrm{~s}, 40$ $\mathrm{mW} / \mathrm{mm}^{2}$ light stimulation on the $\mathrm{Ca}^{2+}$ in mOPN4L-expressing HEK 293T cells within $10 \mu \mathrm{M}$ ATR $(\mathrm{N}=12$ cells; red line) and the lack of such effect following ATR removal (black line). 

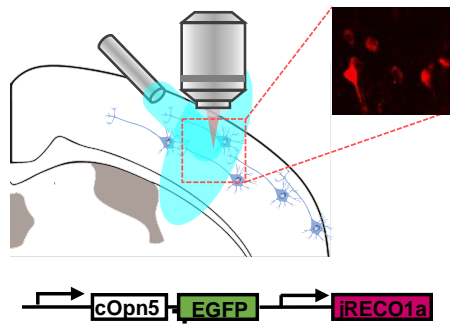

d

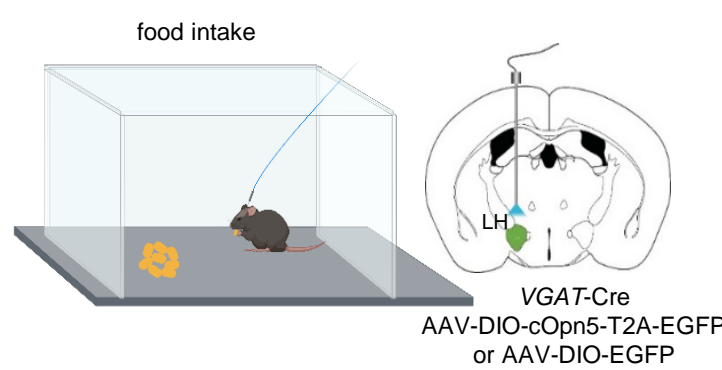

f

high fat food foraging

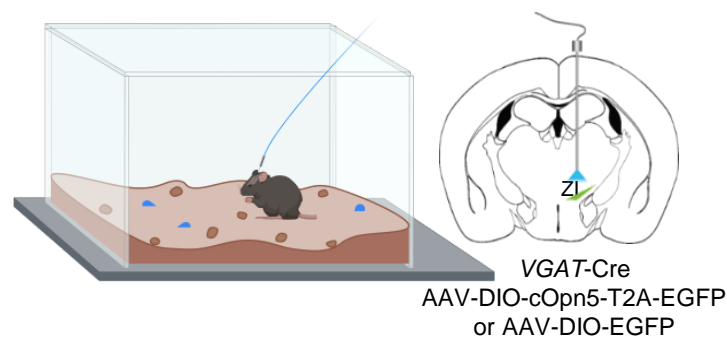

h
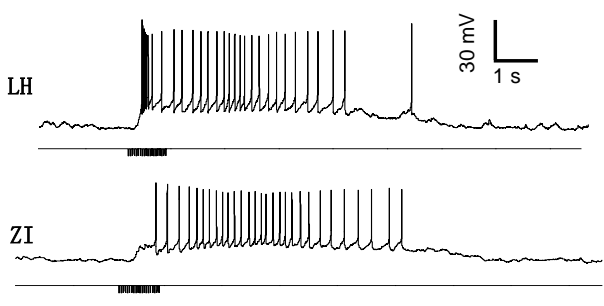

$1 \mathrm{~s}, 20 \mathrm{~Hz}, 10 \mathrm{~ms} /$ pulse
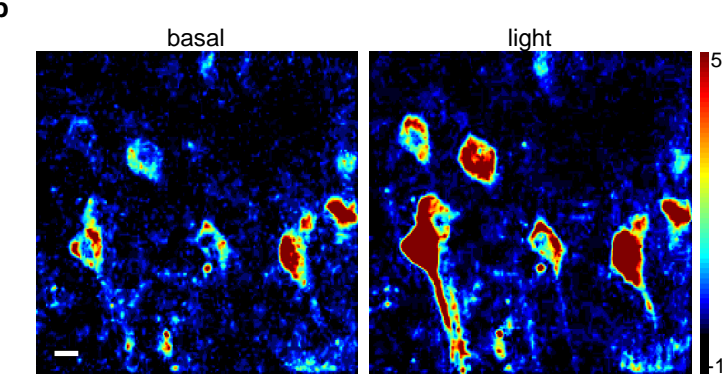

C
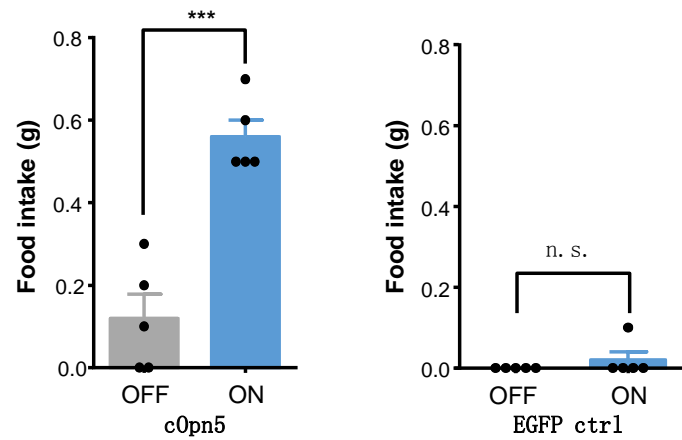

g
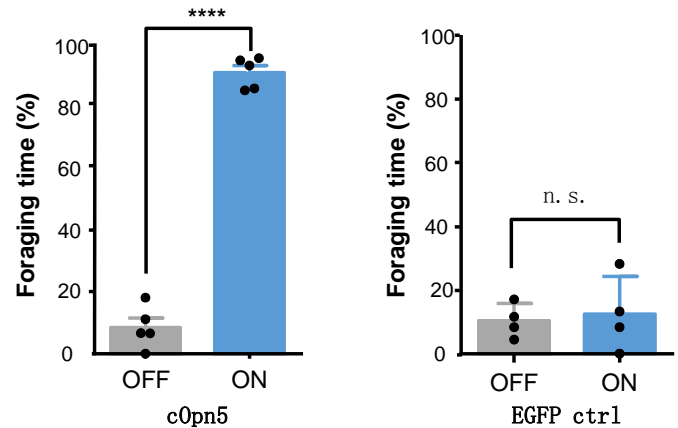

i
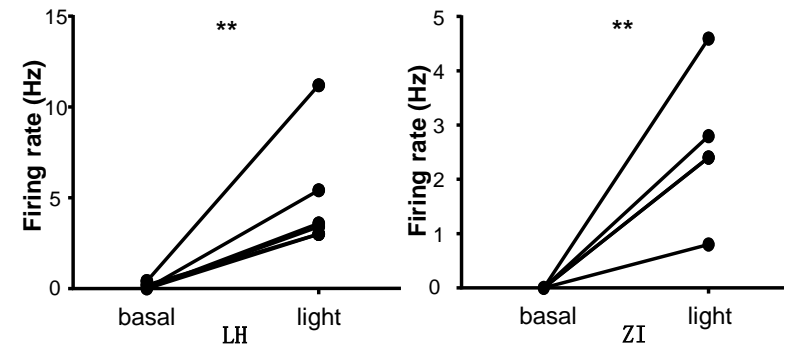
Fig. 3: copn5-mediated optogenetics activation of neurons and changes of mouse behaviors in a neural circuit-dependent manner.

a, Schematic diagram shows the experimental setup for optogenetic stimulation and $\mathrm{Ca}^{2+}$ imaging. We used AAV vectors to express cOpn5, EGFP, and the red $\mathrm{Ca}^{2+}$ sensor jRECO1a in neurons.

b, Pseudocolor images show $\mathrm{Ca}^{2+}$ signals before and after light stimulation $\left(10 \mathrm{~s} ; 100 \mu \mathrm{W} / \mathrm{mm}^{2}\right.$; $473 \mathrm{~nm})$. Scale bar, $10 \mu \mathrm{m}$.

c, Group data of $\mathrm{Ca}^{2+}$ signal traces of 6 individual neurons shown in $\mathbf{b}$.

d, Schematic diagram of the experimental setup for cOpn5 optogenetics and food intake assay. cOpn5 and EGFP were expressed in GABAergic neurons within the LH of VGAT-Cre mice. EGFP was expressed as a control.

e, Summary data show the light-induced $(20 \mathrm{~Hz} ; 5 \mathrm{~ms} /$ pulse; $473 \mathrm{~nm} ; 0.75 \mathrm{~mW}$ output from the fiber tip), cOpn5-mediated activation of eating behaviors. ${ }^{* *} \mathrm{P}=0.0003$; n.s., non-significant; $\mathrm{N}=6$ mice; unpaired $t$ test. Error bars indicate S.E.M..

f, Schematic diagram of the experimental setup for food foraging behavior. High-fat food pellets were used. cOpn5 and EGFP were expressed in GABAergic neurons within the ZI of VGAT-Cre mice. EGFP was expressed as a control.

g. Summary data show the cOpn5-mediaed food foraging behaviors. Foraging time percentage was calculated upon receiving light stimulation until the mouse found the hidden food. ${ }^{* \star *} P$ $<0.0001 ; \mathrm{N}=6$ mice; unpaired $t$ test. Error bars indicate S.E.M..

$\mathbf{h}$, Raw data illustrate the pattern of cOpn5-mediated optical activation of GABAergic neurons in these two brain areas.

i, Group data show the neuronal firing rates before and after pulsed $473 \mathrm{~nm}$ light stimulation $(1 \mathrm{~Hz}$, $5 \mathrm{~s}$; for the $\mathrm{LH}, \mathrm{N}=6$ neurons, ${ }^{\star \star} \mathrm{P}=0.0041$, unpaired t tests; for the $\mathrm{ZI}, \mathrm{N}=5$ neurons, ${ }^{\star \star} \mathrm{P}=$ 0.0027, unpaired t tests). 
(which was not certified by peer review) is the author/funder, who has granted bioRxiv a license to display the preprint in perpetuity. It is $m$ available under aCC-BY-NC-ND 4.0 International license.

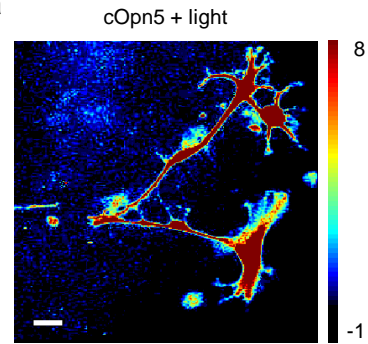

d

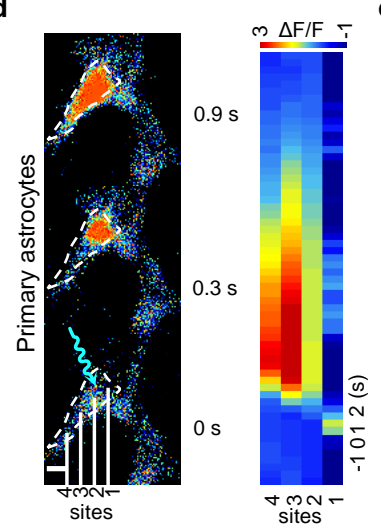

。

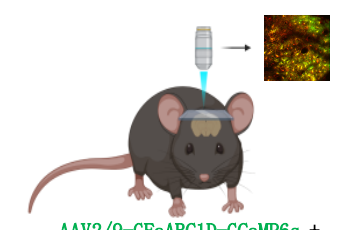

AV2/8-GFaABC1D-c0pn5-T2A-mCherry b
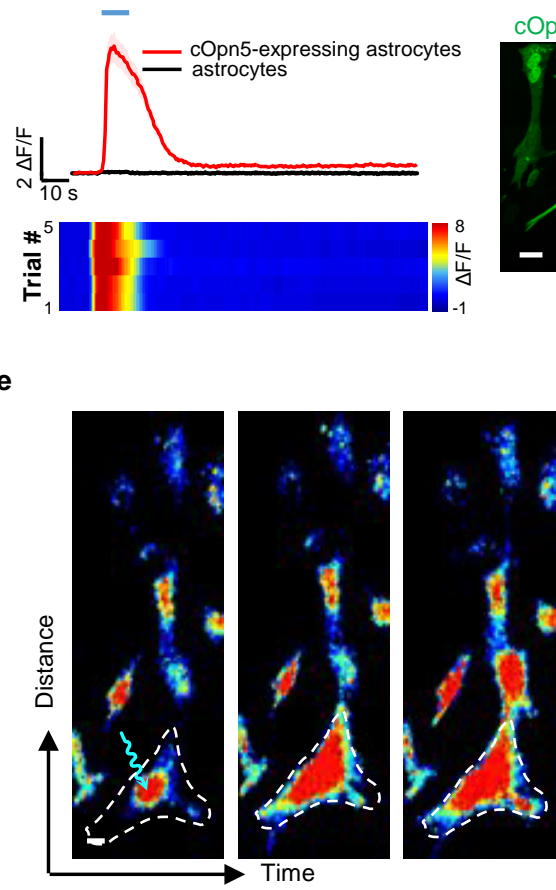

h
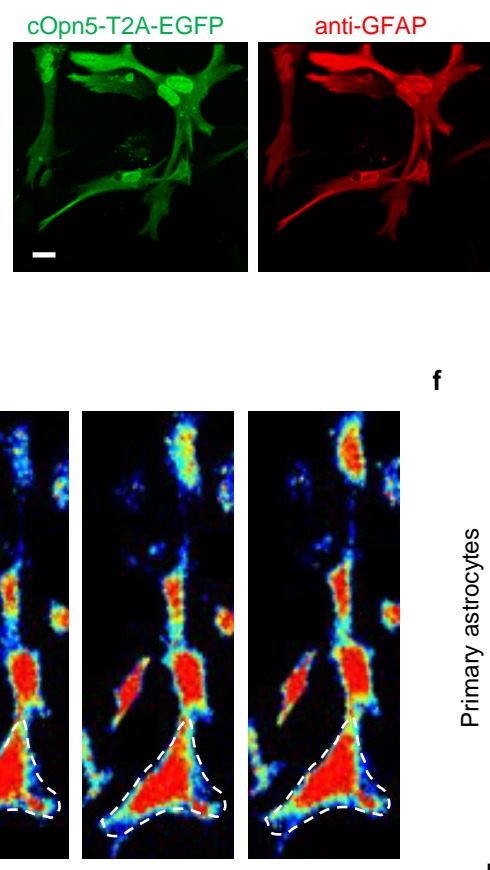

f

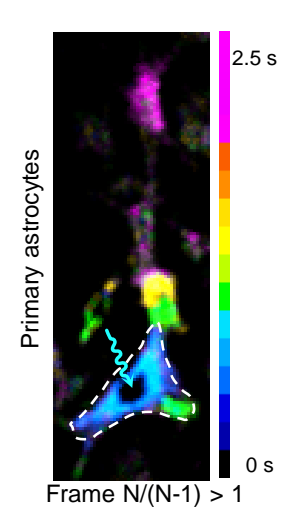

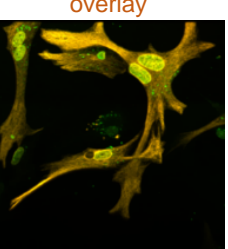

Frame $\mathrm{N} /(\mathrm{N}-1)>1$
cOpn5

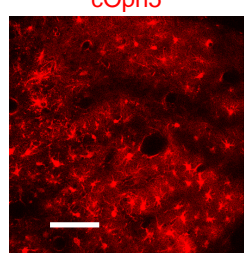

GCaMP6s

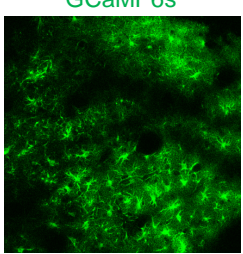

Overlay
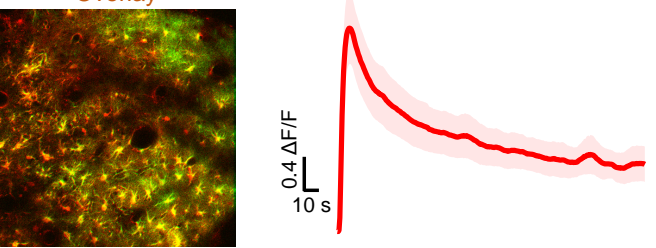

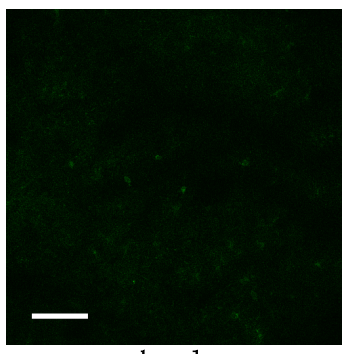

basal

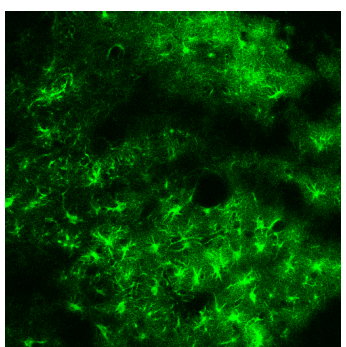

light

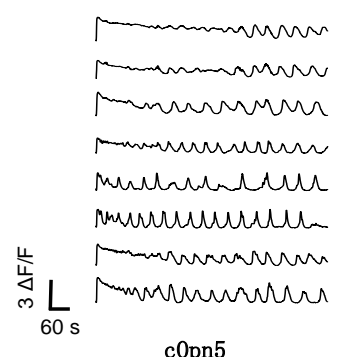

Mh

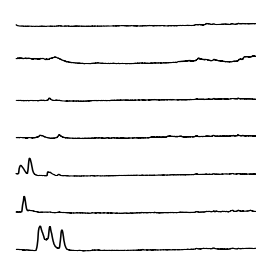

mCherry ctrl 
Fig. 4 cOpn5-mediated optical activation of astrocytes evokes calcium signal.

a, Pseudocolor images of the baseline and peak $\mathrm{Ca}^{2+}$ signals following light stimulation of cOpn5expressing astrocytes. Scale bar, $20 \mu \mathrm{m}$.

b, Plot of $\mathrm{Ca}^{2+}$ signals and heat map representation of $\mathrm{Ca}^{2+}$ signals across trials ( $\mathrm{n}=25$ astrocytes).

c, cOpn5 was expressed in cultured primary astrocytes using AAV-cOpn5-T2A-EGFP (green). Astrocyte identity was confirmed by GFAP immunostaining (red). Scale bar, $20 \mu \mathrm{m}$.

d, Images of light-induced $\mathrm{Ca}^{2+}$ signal propagation in a single cOpn5-expressing primary astrocyte stimulated in a subcellular region (stimulation size $4 \times 4 \mu \mathrm{m}^{2}$ and frame interval $=300 \mathrm{~ms}$ ). Scale bar, 10 $\mu \mathrm{m}$.

e, Images of light-induced $\mathrm{Ca}^{2+}$ signal propagation in cOpn5-expressing primary astrocytes. Scale bar, $10 \mu \mathrm{m}$.

f, Pseudocolor images showing the process of $\mathrm{Ca}^{2+}$ signal propagation across time of $\mathbf{g}$ (frame $\mathrm{N} /(\mathrm{N}-1)$ $>1$ ). Frame interval was $500 \mathrm{~ms}$ and each frame is counted once.

g. Schematic diagram of the experimental setup for in vivo two-photon imaging $(920 \mathrm{~nm})$ of astrocyte $\mathrm{Ca}^{2+}$ imaging following cOpn5-mediated astrocyte activation. Images show the expression of GCaMP6s (green) and cOpn5-T2A-mCherry (red) in astrocytes within the mouse S1 cortex. Scale bar, $100 \mu \mathrm{m}$.

$\mathbf{h}$, Time courses of light-evoked $\mathrm{Ca}^{2+}$ signals in astrocytes $(\mathrm{N}=5)$.

i, Images of the $\mathrm{Ca}^{2+}$ signals before (basal) and 5s after light stimulation (light). Scale bar, $100 \mu \mathrm{m}$.

j, Raw traces of eight individual GCaMP6s-expressing astrocytes signals in a mouse that expressed cOpn5 or mCherry. 
(which was not certified by peer review) is the author/funder, who has granted bioRxiv a license to display the preprint in perpetuity. It is $m$ available under aCC-BY-NC-ND 4.0 International license.

a

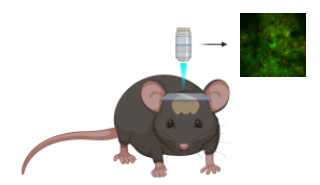

AAV2/9-GFaABC1D-GRAB AAV2/8-GFaABC1D-c0pn5-T2A-mCherry

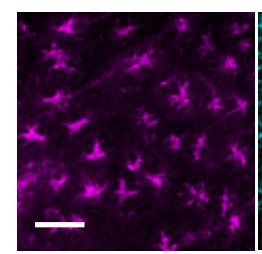

GFAP

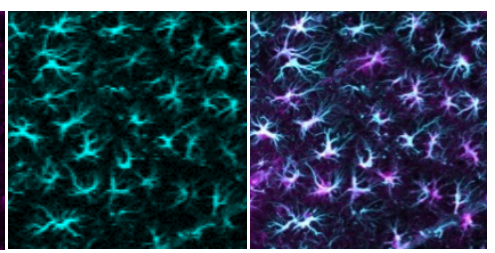

C

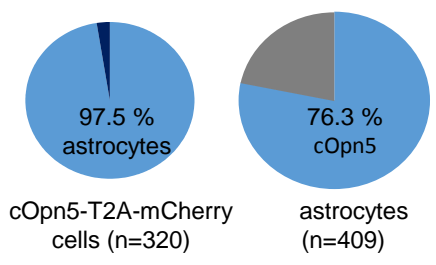

d
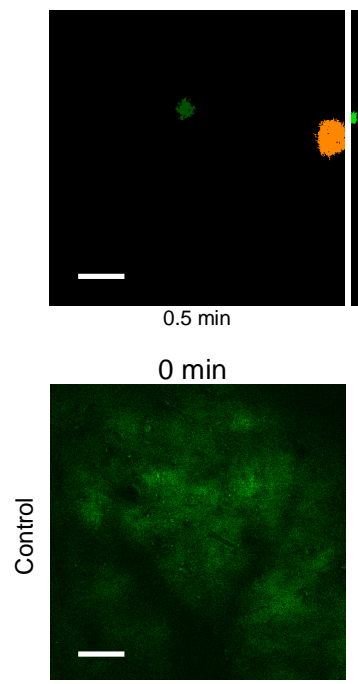

ํํำ
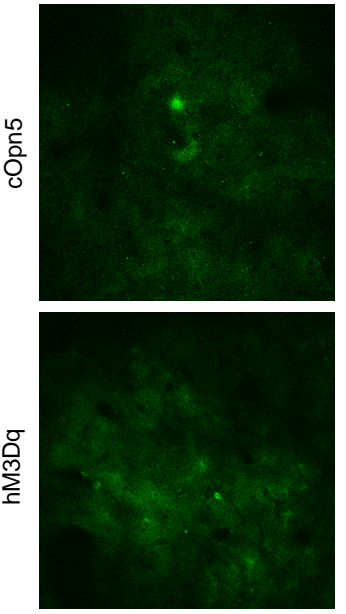

h

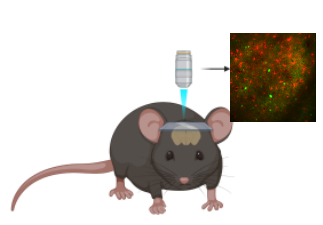

AAV2/9-CaMKII-GCaMP7b +

AAV2/8-GFaABC1D-c0pn5-T2A-mCherry

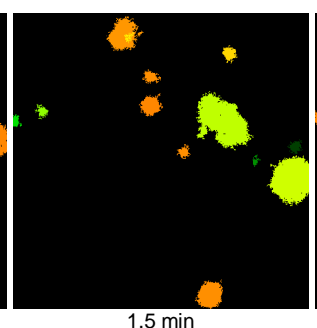

$5 \mathrm{~min}$
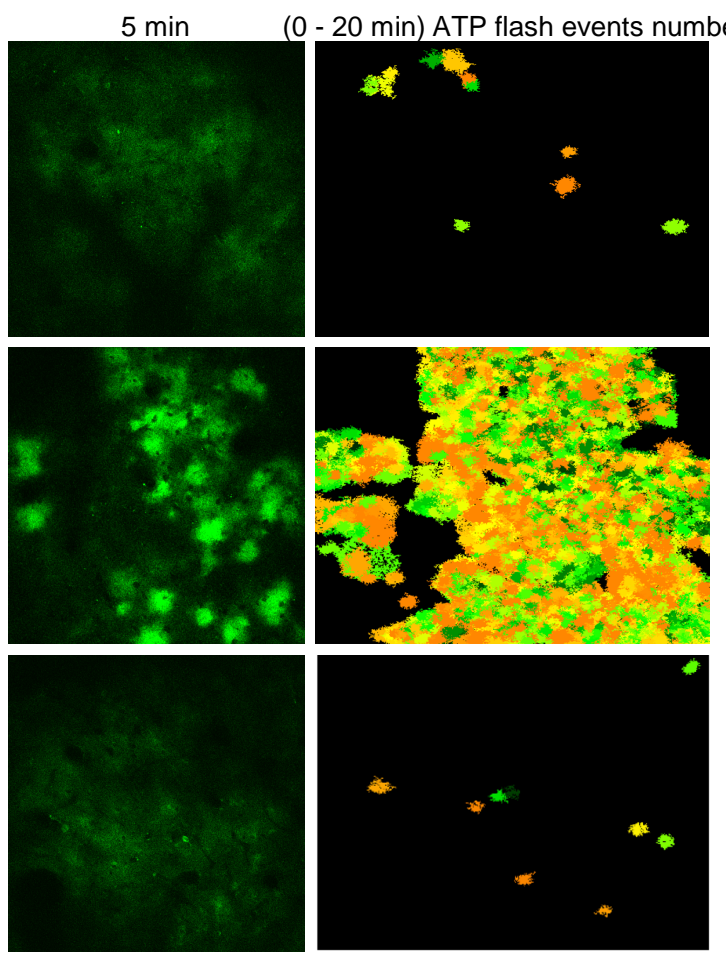

5 min

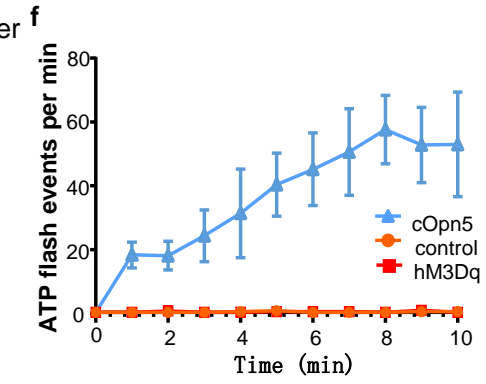

g

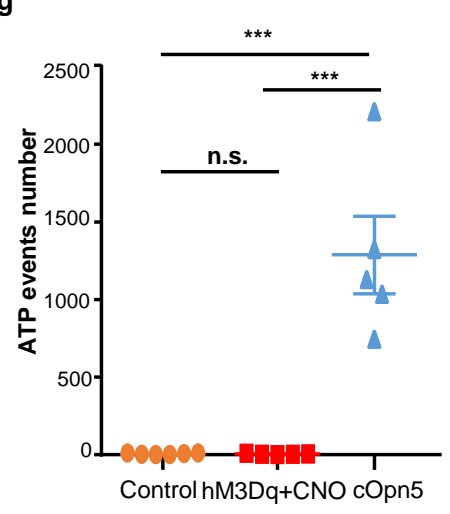

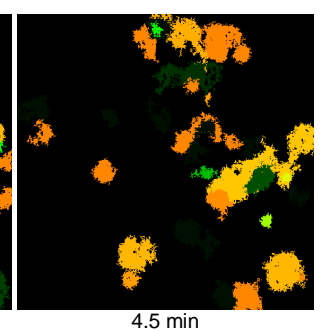
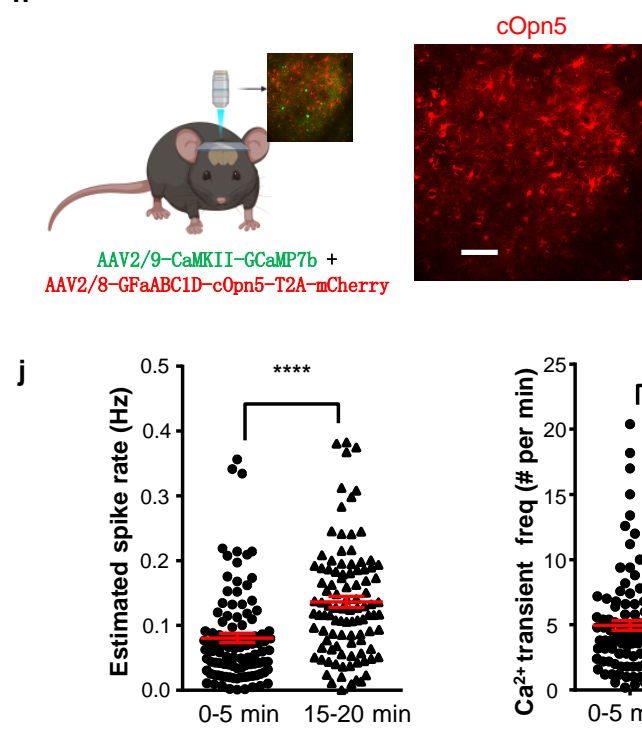

\section{GCaMP7b}

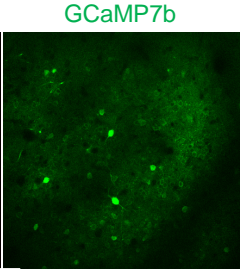

Overlay

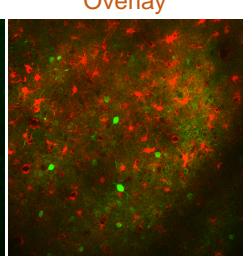

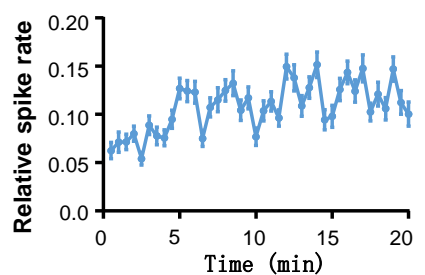

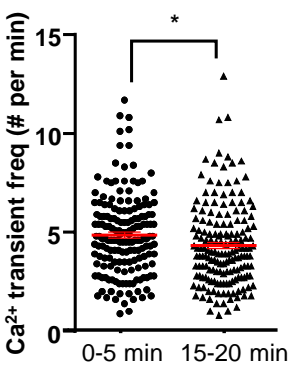

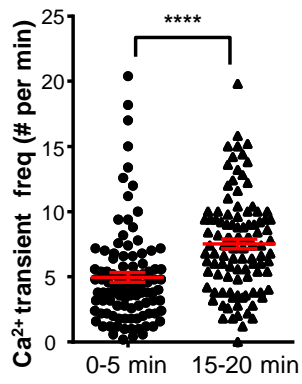

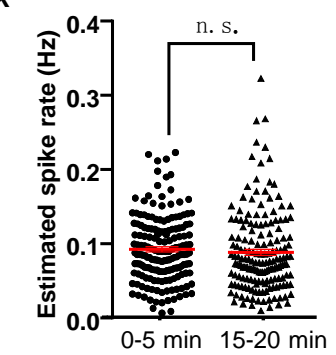


Fig. 5: cOpn5-mediated optogenetic activation of astrocytes induces massive ATP flashes and neuronal activation in vivo.

a, Schematic diagram of the experimental setup for in vivo two-photon imaging (920 nm) of ATP signal following cOpn5-mediated optical activation of astrocytes. We infused two AAV vectors to express cOpn5-T2A-mCherry (red), and GRAB $_{\text {ATP }}$ sensor (green) in the astrocytes within the mouse S1 cortex.

b, Pseudocolor images show that AAV-GfaABC1D-cOpn5-T2A-mCherry drove mCherry expression in S1 cortical cells that were immunopositive to GFAP, a marker of astrocytes. Scale bar, $20 \mu \mathrm{m}$.

c, Out of 320 cOpn5-mCherry+ cells examined, 312 cells were GFAP+, indicating $97.5 \%$ precision. Within the same imaged areas, a total of 409 cells were GFAP, indicating $76.3 \%$ efficiency of astrocyte labeling using the AAV-GFaABC1D constructs.

d, Representative images show astrocytic ATP flash events in a cOpn5-expressing mouse in the first 4.5min, with different colors indicating individual flashes. Scale bar, $100 \mu \mathrm{m}$.

e, Images show the overall ATP flash events in a control mouse (no cOpn5 expression), a CNO (2 $\mathrm{mg} / \mathrm{kg}$ ) - treated hM3Dq expression mouse (no cOpn5 expression) and a cOpn5-expressing mouse. The left column shows the raw $\mathrm{GRAB}_{\mathrm{ATP}}$ images at the basal level (before light delivery); the middle column shows GRAB $_{\text {ATP }}$ signals at $5 \mathrm{~min}$; and the right column shows pseudo-color-coded ATP flash events accumulated during 0-20 min. Scale bar, $100 \mu \mathrm{m}$.

f, The plot of astrocytic ATP flash events number per min across time (0-10 min) in control group (no cOpn5 expression), CNO-treated hM3Dq expression group (no cOpn5 expression), and cOpn5-expressing group.

g. The quantification of ATP flash events number in 20min of the control $(N=6), h M 3 D q+C N O(N$ $=5)$, and cOpn5 groups $(\mathrm{N}=5)$. For the control - hM3Dq+CNO comparison, no significant difference; for the control - cOpn5 comparison, ${ }^{\star * *} \mathrm{P}=0.0003$, for the $\mathrm{hM} 3 \mathrm{Dq}+\mathrm{CNO}-\mathrm{cOpn5}$ comparison, ${ }^{\star \star *} \mathrm{P}=0.0009$; unpaired $t$ tests.

$\mathbf{h}$, Schematic diagram of the experimental setup for in vivo two-photon imaging $(920 \mathrm{~nm})$ of neuron $\mathrm{Ca}^{2+}$ imaging following cOpn5-mediated astrocyte activation. Images show the expression of cOpn5-T2A-mCherry (red) in astrocytes and the expression of a GCaMP7b (green) in neurons within the mouse S1 cortex. Scale bar, $100 \mu \mathrm{m}$.

i, The plot of average spike rate relative to basal across time $(0-20 \mathrm{~min}) . \mathrm{N}=193$ neurons from 3 mice

j, Decoded spike rate analysis of GCaMP7b-expressing neurons within the periods of 0-5 min and 15-20 min that were coupled with cOpn5-mediated astrocytes activation (left panel) $\mathrm{N}=193$ neurons). ${ }^{\star \star \star \star} \mathrm{P}<0.0001$, unpaired $t$ test. The number of $\mathrm{Ca}^{2+}$ transients of GCaMP7b-expressing neurons during 0-5 min and 15-20 min, coupled with cOpn5-mediated astrocytes activation (right panel). $\mathrm{N}=193$ neurons, ${ }^{\star \star \star *} \mathrm{P}<0.0001$, Unpaired $t$ test.

$\mathbf{k}$, Decoded spike rate analysis of GCaMP7b-expressing neurons within the periods of 0-5 min and 15-20 min that were coupled with imaging from mice with mCherry expression in astrocytes as control (left panel) ( $\mathrm{N}=170$ neurons). no significant difference; unpaired $t$ test. The number of $\mathrm{Ca}^{2+}$ transient analysis of GCaMP7b-expressing neurons during the periods of 0-5 min and 15-20 min in mice that expressed mCherry in astrocytes (right panel). $\mathrm{N}=170$ neurons, ${ }^{*} \mathrm{P}=0.0146$, paired $t$ test. 


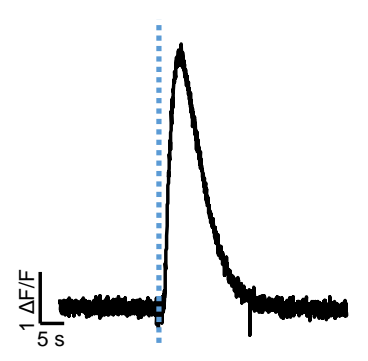

d

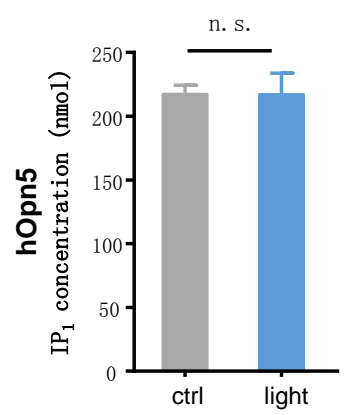

b

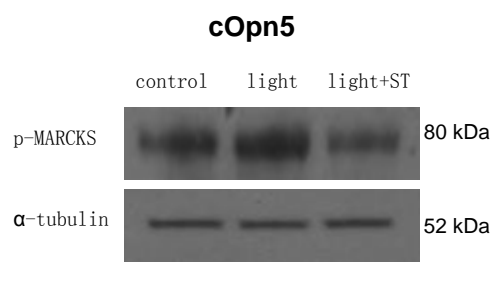

e

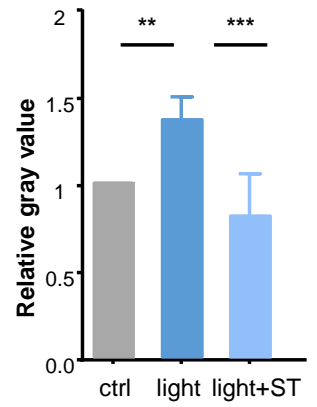

\section{Extended Data Fig. 1 cOpn5 couples to $G_{q}$ signaling.}

a, Time course of optically evoked $\mathrm{Ca}^{2+}$ signal in extracellular $\mathrm{Ca}^{2+}$ free medium (10 ms; 16 $\mu \mathrm{W} / \mathrm{mm}^{2} ; 470 \mathrm{~nm} ; \mathrm{N}=17$ HEK 293T cells).

b, One representative of phosphorylation of MARCKS in cOpn5-expressing HEK 293T cells in the control group (no light stimulation), the light stimulation group, and light+staurosporine (ST) group (ST, a PKC inhibitor; $10 \mu \mathrm{M}$ ) without addition of retinal.

c, The amount of $\mathrm{p}$-MARCKS was normalized to the amount of $\alpha$-tubulin in the same fraction. $\mathrm{N}=4$, ${ }^{\star *} \mathrm{P}=0.0096,{ }^{\star \star *} \mathrm{P}=0.0004$; Tukey's multiple comparisons test.

d, $\mathrm{IP}_{1}$ accumulation in human Opn5-expressing HEK 293T cells with or without light stimulation without addition of retinal. $\mathrm{N}=4$, n.s., no significant difference; unpaired $t$ test.

e, Light has no effect on cAMP levels (10 $\mu \mathrm{M}$ forskolin preincubation) in cOpn5-expressing HEK 293T cells without additional retinal in the medium (left panel) $(N=4)$. Right panel shows the effects of photostimulation on cAMP concentrations for HEK 293T cells expressing Opn5s from four different species following $10 \mu \mathrm{M}$ retinal Preincubation $(\mathrm{N}=4)$. n.s., no significant difference; unpaired $t$ test.

Error bars in $\mathbf{c}, \mathbf{d}$ and $\mathbf{e}$ indicate S.E.M.. 
a

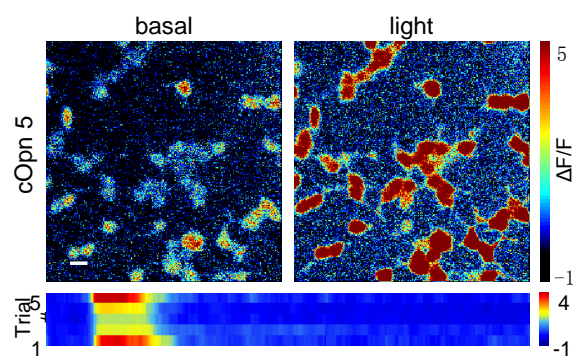

b

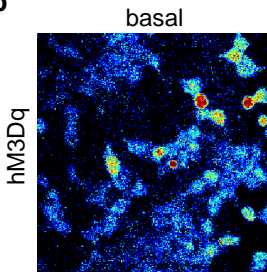

CNO

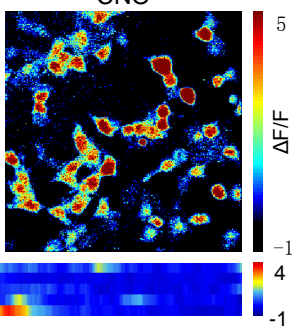

c

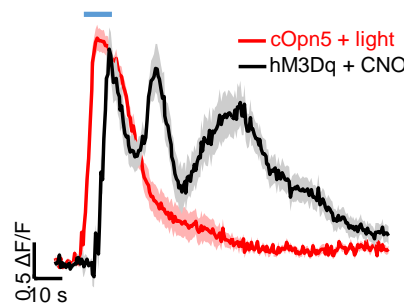

Extended Data Fig. 2 Direct comparisons between the performance of cOpn5 and hM3Dq chemogenetics.

a Pseudocolor images (upper) and heat map representation of $\mathrm{Ca}^{2+}$ signals evoked by $10 \mathrm{~s}$ optical stimulation (same as the CNO stimulation time) of cOpn5-expressing cells across 5 consecutive trials. Scale bar, $20 \mu \mathrm{m}$.

b, Effect of chemogenetic stimulation on the $\mathrm{Ca}^{2+}$ signals in hM3Dq-expressing HEK 293T cells. c, Time courses of $\mathrm{Ca}^{2+}$ signals evoked by cOpn5-mediated optogenetic stimulation (10 s) and hM3Dq-mediated chemogenetic stimulation using CNO puff $(100 \mathrm{nM} ; 10 \mathrm{~s})$, respectively $(\mathrm{N}=$ 20, cOpn5-expressing HEK 293T cells and 20 hM3Dq-expressing HEK 293T cells). 
a

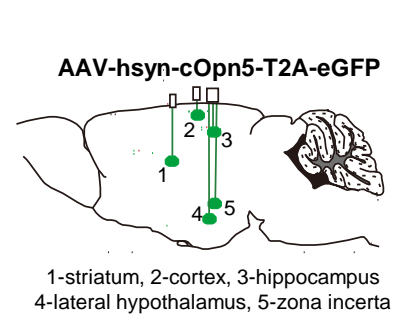

b

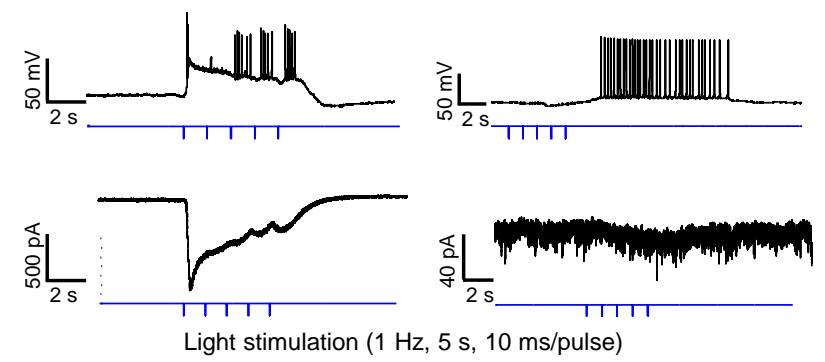

C

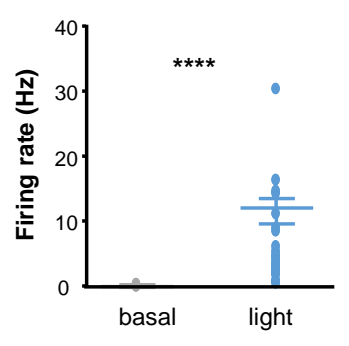

d

\begin{abstract}
$10 \mathrm{~Hz}, 5 \mathrm{~s}, 10 \mathrm{~ms} /$ pulse
\end{abstract}
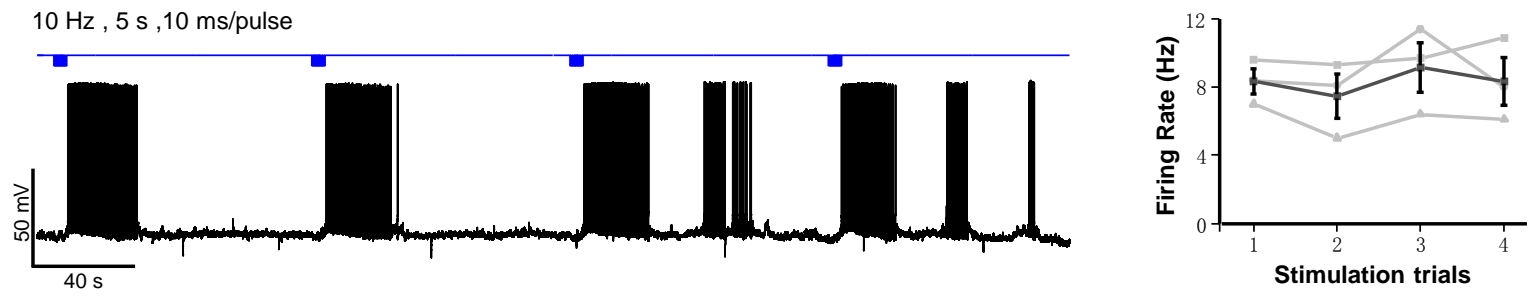

e
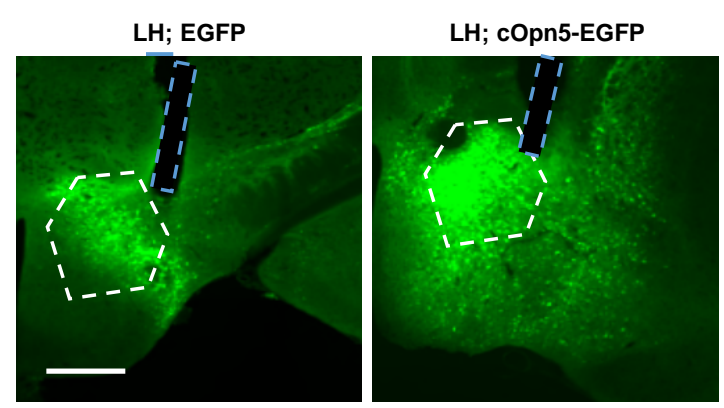

f
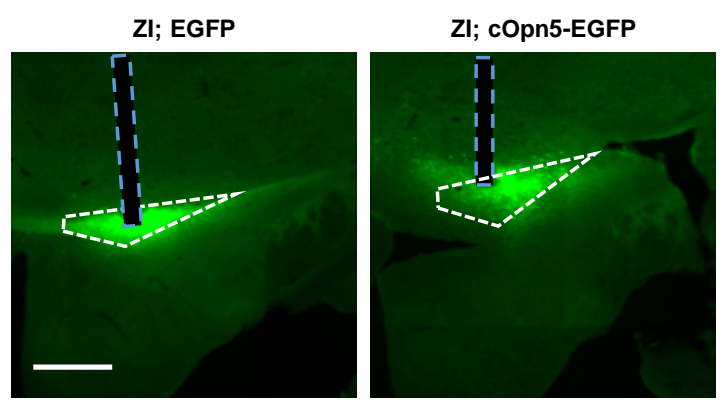

Extended Data Fig. 3 copn5-mediated optogenetics reliably activates neurons.

a, Schematic diagram depicts optogenetic stimulation and whole-cell patch-clamp recording of cOpn5-expressing neurons in the cortex, striatum, hippocampus, lateral hypothalamus (LH), and zonal incerta (ZI).

b, Raw data illustrate two representative patterns of light-evoked neuronal activation. One neuron (left panels) exhibited rapid membrane potential depolarization and large inward currents $(1 \mathrm{~Hz}, 5 \mathrm{~s}$, $10 \mathrm{~ms} /$ pulse), and another neuron exhibited strong, delayed firing of action potentials yet small sustained inward currents in response to the light pulses.

c, Group data show the neuronal firing rates before and after pulsed $473 \mathrm{~nm}$ light stimulation (20 $\mathrm{Hz}, 1 \mathrm{~s} ; \mathrm{N}=29$ neurons; $* \star \star \star \mathrm{P}<0.0001$, unpaired $t$ test).

d, Raw trace shows that copn5 mediated reliable and reproducible photoactivation of a neuron (left). The right panel shows the summary of firing rates across repetitive trials of light stimulation ( $N=3$ neurons).

e, Images show the expression of EGFP control and bicistronic expression of EGFP and cOpn5 in the LH (white dashed lines). Lesion sites and blue dashed lines indicate the placement of optical fibers. Scale bars, $500 \mu \mathrm{m}$.

f, The injection sites and optical fiber placement in the ZI. Scale bars, $500 \mu \mathrm{m}$. 
a

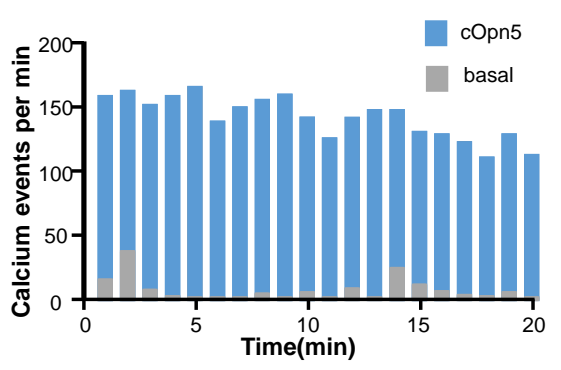

c

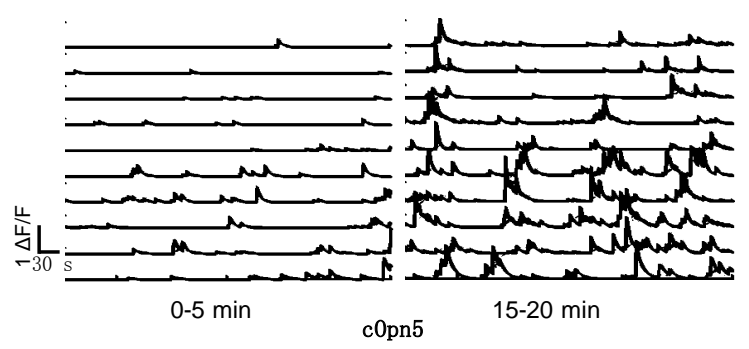

b

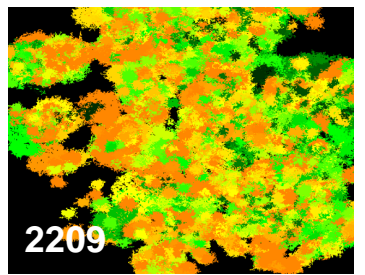

c0pn5

First trial 0-20 min

d

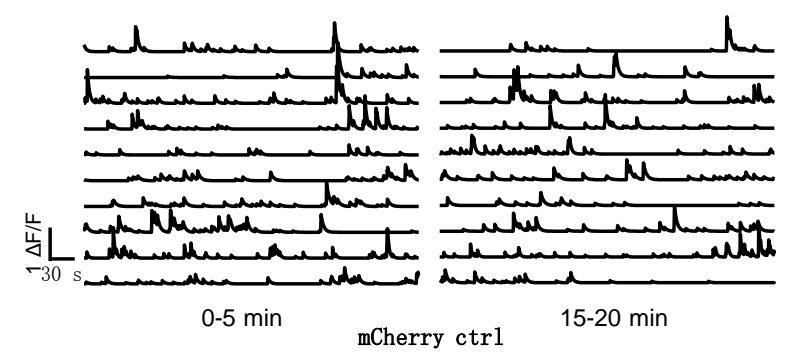

Extended Data Fig. 4 cOpn5-mediated optical activation of astrocytes, produces reliable ATP release and the activation of surrounding neurons.

a, Time courses of light-evoked $\mathrm{Ca}^{2+}$ events in astrocytes for $20 \mathrm{~min}$ constant stimulation $(\mathrm{N}=5)$.

b, Pseudocolor color image show total ATP flash events (0-20 $\mathrm{min})$ in cOpn5-expressing mouse in a repeat trial 1 hour after the initial trial.

c, Raw traces of ten individual GCaMP7b-expressing neurons signals in 0-5 min and 15-20 min, coupled with copn5-mediated optical activation of astrocytes .

d, Raw traces of ten individual GCaMP7b-expressing neurons signals during the periods of $0-5 \mathrm{~min}$ and 15-20 min in a mouse that expressed mCherry in astrocytes. 